\title{
Cellular approximations and the Eilenberg-Moore spectral sequence
}

\author{
SHOHAM SHAMIR
}

\begin{abstract}
We set up machinery for recognizing $k$-cellular modules and $k$-cellular approximations, where $k$ is an $R$-module and $R$ is either a ring or a ring-spectrum. Using this machinery we can identify the target of the Eilenberg-Moore cohomology spectral sequence for a fibration in various cases. In this manner we get new proofs for known results concerning the Eilenberg-Moore spectral sequence and generalize another result.
\end{abstract}

$55 \mathrm{P} 43,55 \mathrm{~T} 20$

\section{Introduction}

Given a pointed topological space $A$, the $A$-cellularization of another pointed space $X$ is the closest approximation of $X$ built from $A$ using pointed homotopy colimits. This notion of $A$-cellularization was first treated systematically by Dror Farjoun [3] and Chachólski [2]. Also fundamental to this subject is the work of Hirschhorn [12]. Dwyer and Greenlees translated this concept to an algebraic setting in [6]. Further work on cellular approximation was done by Dwyer, Greenlees and Iyengar in [7], here in the wider setting of $\mathbb{S}$-algebras, where $\mathbb{S}$ is the sphere spectrum.

The algebraic setting of Dwyer and Greenlees from [6] is as follows. Let $R$ be a ring (with a unit, not necessarily commutative) and let $k$ and $C$ be $\mathbb{Z}$-graded chain complexes of left $R$-modules. We say $C$ is $k$-cellular over $R$, if $\operatorname{ext}_{R}^{*}(k, N)=0$ implies $\operatorname{ext}_{R}^{*}(C, N)=0$ for every $R$-chain complex $N$. A $k$-cellular approximation of a complex $X$ is a map of $R$-complexes $\operatorname{Cell}_{k}^{R} X \rightarrow X$ such that $\operatorname{Cell}_{k}^{R} X$ is the closest approximation of $X$ by a $k$-cellular complex in the sense that the induced map $\operatorname{ext}_{R}^{*}\left(k, \operatorname{Cell}_{k}^{R} X\right) \rightarrow \operatorname{ext}_{R}^{*}(k, X)$ is an isomorphism.

The concept of $k$-cellular approximation extends effortlessly to the stable homotopy category, which is the setting used here. Specifically, we let $R$ be an $\mathbb{S}$-algebra in the sense of Elmendorf, Kriz, Mandell and May [10], where $\mathbb{S}$ denotes the sphere spectrum. Let $k$ and $C$ be left $R$-modules. Then the definitions above immediately apply also to the category of left $R$-modules. 
Dwyer, Greenlees and Iyengar gave in [7] a formula for $k$-cellular approximation. This formula holds when $k$ is a proxy-small $R$-module (see Definition 2.5); in such a case the $k$-cellular approximation of an $R$-module $X$ is given by the following natural map [7, Theorem 4.10]:

$$
\operatorname{Hom}_{R}(k, X) \otimes_{\operatorname{End}_{R}(k)} k \rightarrow X
$$

The symbol $\otimes_{R}$ denotes the smash product of $R$-modules and $\operatorname{Hom}_{R}(-,-)$ stands for the function spectrum of $R$-modules. These functors are taken in the derived sense, ie we always take appropriate resolutions before applying them.

Dwyer, Greenlees and Iyengar's formula for $k$-cellular approximations can be used to understand the target of the Eilenberg-Moore cohomology spectral sequence for a fibration, as we will now demonstrate. Suppose $k$ is a commutative $\mathbb{S}$-algebra and let $F \rightarrow E \rightarrow B$ be fibration of spaces where $E$ and $B$ are connected. We make the following standard replacements. First, one can replace $\Omega B$ by an equivalent topological group, so we will assume $\Omega B$ is a topological group. Second, standard constructions show we can replace $F$ by an equivalent $\Omega B$-space (by passing, perhaps, to an equivalent fibration) so we will assume that $F$ itself is a right $\Omega B$-space. Let $\mathrm{C}^{*}(F ; k)$ denote the function spectrum $F_{\mathbb{S}}\left(\Sigma^{\infty} F_{+}, k\right)$ and let $k[\Omega B]$ denote the $\mathbb{S}-$ algebra $k \wedge_{\mathbb{S}} \Sigma^{\infty} \Omega B_{+}$. Thus $C^{*}(F ; k)$ is a left $k[\Omega B]$-module. Following [7], we shall henceforth use the notation $\otimes$ for the smash product of spectra.

The same arguments of Dwyer and Wilkerson from [9, Lemma 2.10] can be used to show there is a weak equivalence of $k$-modules:

$$
\operatorname{Hom}_{k[\Omega B]}\left(k, \mathrm{C}^{*}(F ; k)\right) \otimes_{\operatorname{End}_{k[\Omega B]}(k)} k \simeq \mathrm{C}^{*}(E ; k) \otimes_{\mathrm{C}^{*}(B ; k)} k
$$

On the other hand, recall the Eilenberg-Moore cohomology spectral sequence for this fibration (with coefficients in $k$ ) has the form:

$$
E_{p, q}^{2}=\operatorname{tor}_{p, q}^{H^{*}(B ; k)}\left(H^{*}(E ; k), k\right) \Rightarrow \pi_{p+q}\left(\mathrm{C}^{*}(E ; k) \otimes_{\mathrm{C}^{*}(B ; k)} k\right)
$$

Thus, whenever $k$ is a proxy-small $k[\Omega B]$-module, the Eilenberg-Moore cohomology spectral sequence computes the homotopy groups of the $k$-cellular approximation of $\mathrm{C}^{*}(F ; k)$ over $k[\Omega B]$. In particular, if $\mathrm{C}^{*}(F ; k)$ is a $k$-cellular $k[\Omega B]-$ module, then the target of the Eilenberg-Moore cohomology spectral sequence is the cohomology of the $F$ with coefficients in $k$.

Using machinery for recognizing $k$-cellular modules and $k$-cellular approximations we get the following two theorems. 
Theorem 5.1 Fix a prime $p$. Let $N$ be a finite nilpotent group and let $P \subseteq N$ be the $p$-Sylow subgroup of $N$, so $N \cong P \times H$ with the order of $H$ being prime to $p$. Let $F \rightarrow E \rightarrow \mathrm{B} N$ be a homotopy fibration sequence over the classifying space of $N$, with $E$ being a connected space. If $k$ is any commutative $\mathbb{S}$-algebra such that $\pi_{0}(k)$ is an $\mathbb{F}_{p}$-algebra, then

$$
\mathrm{C}^{*}(E ; k) \otimes_{\mathrm{C}^{*}(B ; k)} k \simeq \mathrm{C}^{*}\left(F_{h(H)} ; k\right)
$$

where $F_{h(H)}$ is the homotopy orbit space of $F$ with respect to the $H$-action on $F$. In particular, if $N=P$ then:

$$
\mathrm{C}^{*}(E ; k) \otimes_{\mathrm{C}^{*}(B ; k)}=\mathrm{C}^{*}(F ; k)
$$

Theorem 5.2 Fix a prime $p$. Let $B$ be a finite connected nilpotent space with a finite fundamental group $N=\pi_{1}(B)$. Let $P \subseteq N$ be the $p$-Sylow subgroup of $N$, so $N \cong P \times H$. Let $F \rightarrow E \rightarrow B$ be a homotopy fibration sequence over $B$, where $E$ is a connected space. Then

$$
\operatorname{tor}_{-n}^{\mathrm{C}^{*}\left(B ; \mathbb{F}_{p}\right)}\left(\mathrm{C}^{*}\left(E ; \mathbb{F}_{p}\right), \mathbb{F}_{p}\right)=H^{n}\left(F ; \mathbb{F}_{p}\right)^{H}
$$

where $H^{n}\left(F ; \mathbb{F}_{p}\right)^{H}$ are the fixed points of the $H$-action on $H^{n}\left(F ; \mathbb{F}_{p}\right)$.

The first theorem deals with general multiplicative cohomology theories in characteristic $p$ and thus generalizes a result of Kriz from [13], showing convergence of the Eilenberg-Moore cohomology spectral sequence for a fibration over $\mathrm{B}(\mathbb{Z} / p)$, with coefficients in Morava $K$-theories. The second result concerns only mod- $p$ cohomology. This result is hardly surprising as it is dual to Dwyer's exotic convergence [5], which concerns convergence of the Eilenberg-Moore homology spectral sequence. However, the proof we give here uses only cellularity arguments. We also give a different proof for a weaker version of Dwyer's strong convergence result from [4], again using only cellularity arguments. This is done in Proposition 5.8.

Another result is a spectral sequence. Assuming that $k$ is a commutative ring, we describe in Section 6 a spectral sequence with

$$
E_{p, q}^{1}=\pi_{2 p+q}\left(\operatorname{Cell}_{k}^{k\left[\pi_{1} B\right]} H^{p}(F ; k)\right)
$$

that converges to $\pi_{p+q}\left(\mathrm{Cell}_{k}^{k[\Omega B]} \mathrm{C}^{*}(F ; k)\right)$ when $B$ is a finite nilpotent space. We demonstrate the use of this spectral sequence in Lemma 6.3 and Corollary 6.4.

The bulk of this paper is concerned with setting up the necessary machinery for recognizing $k$-cellular modules and $k$-cellular approximations. This machinery is 
interesting in itself and we mention one basic result. Let $R \rightarrow S$ be a map of $\mathbb{S}-$ algebras and let $k$ be an $S$-module. The independence of base lemma (Lemma 3.1) relates $k$-cellular approximation of $S$-modules with their $k$-cellular approximation as $R$-modules.

Lemma 3.1 Let $R \rightarrow S$ be a map of $\mathbb{S}$-algebras.

(1) (Strong independence of base) Let $k$ be an $S$-module. If $S \otimes_{R} k$ is $k$-cellular as an $S$-module, then for any $S$-module $X$ the map $\operatorname{Cell}_{k}^{S} X \rightarrow X$ is a $k$ cellular approximation of $X$ as an $R$-module. In particular $\operatorname{Cell}_{k}^{R} X \simeq \operatorname{Cell}_{k}^{S} X$ as $R$-modules.

(2) (Weak independence of base) Let $k$ be an $R$-module. If $S \otimes_{R} k$ is $k$-cellular as an $R$-module, then for any $S$-module $X$ the map $\mathrm{Cell}_{S \otimes_{R}}^{S} X \rightarrow X$ is a $k$-cellular approximation of $X$ as an $R$-module. In particular $\operatorname{Cell}_{k}^{R} X \simeq$ Cell ${ }_{S}^{S} \otimes_{R} k$ as $R$-modules.

This lemma is the basis for results on $k$-cellular approximation over a group-ring $k G$, where $G$ is a topological or discrete group and $k G$ denotes the $\mathbb{S}$-algebra $\mathrm{C}_{*}(G ; k)=$ $k \wedge_{\mathbb{S}} \Sigma^{\infty} G_{+}$. See for example Proposition 3.3.

\subsection{Layout of this work}

We begin by introducing the notions of cellularity and cellular approximation in Section 2. There we also recall some basic properties of cellular approximations and give elementary examples.

The necessary machinery for recognizing $k$-cellular modules and building $k$-cellular approximations is set up in Section 3 and Section 4. In Section 3 we prove the independence of base lemma and apply it to get results concerning $k$-cellular approximation over a group ring $k G$. Section 4 deals with $k$-cellular approximation over finite $p$-groups and finite nilpotent groups.

Finally, in Section 5, we come to the Eilenberg-Moore spectral sequence, applying the machinery we constructed earlier to identify its target. Section 6 is devoted to constructing the spectral sequence and demonstrating its use.

\subsection{Setting and conventions}

We work in the category of left $R$-modules where $R$ is an $\mathbb{S}$-algebra in the sense of [10] and $\mathbb{S}$ stands for the sphere spectrum. However, in some instances we will prefer to use an ordinary ring $R$ and work in the category of differential graded left 
$R$-modules (ie $\mathbb{Z}$-graded chain complexes of left $R$-modules). We justify this in the following way. Recall that for a ring $R$, the $\mathbb{S}$-algebra $H R$ is the appropriate Eilenberg-Mac Lane $\mathbb{S}$-algebra. A result of Schwede and Shipley [15, Theorem 5.1.6], shows the model category of $H R$-modules is Quillen equivalent to the projective model category of unbounded chain complexes over $R$. In this way one can pass between $H R$-modules and differential graded $R$-modules without fear and we will usually not distinguish between the two. To keep the terminology consistent, when $R$ is a ring the term $R$-module will mean a differential graded $R$-module and we will refer to the $R$-modules in the classical sense as discrete $R$-modules (this is the same terminology as in [7]).

We follow [7] in both terminology and notation. Thus, as in [7], we use the notation $-\otimes_{R}-$ for the smash product of $R$-modules. Similarly we use $\operatorname{Hom}_{R}(-,-)$ to denote the function spectrum of $R$-modules. All functors in this paper are taken in the derived sense, in particular $-\otimes_{R}-$ and $\operatorname{Hom}_{R}(-,-)$. This means that we always assume to have replaced our modules by appropriate resolutions before applying the functor in question.

Given $R$-modules $k$ and $M$, we denote the $k$-cellular approximation of an $R$ module $M$ by $\operatorname{Cell}_{k}^{R} M$, or by $\operatorname{Cell}_{k} M$ whenever $R$ is clear from the context.

The homotopy groups of an $R$-module $M$ are the stable homotopy groups of its underlying spectrum, and are denoted as usual by $\pi_{i}(M)$. The derived (or homotopy) category of $R$-modules is denoted by $\mathbf{D}_{R}$. Since this is a triangulated category, we sometimes use the term triangle to indicate a homotopy cofibration sequence. If $f: M \rightarrow N$ is a map of $R$-modules, then $\operatorname{Cone}(f)$ denotes the mapping cone of $f$. We say two $R$-modules are equivalent if they are isomorphic in $\mathbf{D}_{R}$, and use the symbol $\simeq$ to denote this. Similarly two maps between $R$-modules are said to be equivalent if they become equal when passing to the derived category $\mathbf{D}_{R}$. An $R-$ module $M$ is called connective if $\pi_{i}(M)=0$ for all $i<0$. We say that $M$ is bounded above (respectively bounded below) if there exists some index $n$ such that $\pi_{i}(M)=0$ for all $i>n$ (respectively $i<n$ ).

If $k$ is a commutative $\mathbb{S}$-algebra and $X$ is a based space, then the chains of $X$ with coefficients in $k$ is the spectrum:

$$
\mathrm{C}_{*}(X ; k)=k \otimes_{\mathbb{S}} \Sigma^{\infty} X
$$

When $k$ is understood from the context we will simply use the notation $\mathrm{C}_{*}(X)$. If $X$ has no base point we add a disjoint base point to $X$ before taking the chains, ie $\mathrm{C}_{*}(X ; k)=k \otimes_{\mathbb{S}} \Sigma^{\infty} X_{+}$. Similarly, the cochains of based space $X$ with coefficients 
in $k$ is the function spectrum:

$$
\mathrm{C}^{*}(X ; k)=\operatorname{Hom}_{\mathbb{S}}\left(\Sigma^{\infty} X, k\right)
$$

All our spaces are assumed to have the homotopy type of CW-spaces. We use pt to denote the space with a single point. For a based space $X$, the space $\Omega X$ is a topological group weakly equivalent to the loop-space of $X$.

In many cases the ring $R$ we work over will be a group-ring. If $G$ is a topological group and $k$ is a commutative $\mathbb{S}$-algebra, the group-ring $k G$ is $\mathrm{C}_{*}(G ; k)$, which is an $\mathbb{S}$-algebra. If $k$ is a commutative ring and $G$ a discrete group, then $H k[G]$ is equivalent to the Eilenberg-Mac Lane spectrum of the usual group-ring $k G$. When $R$ is a group-ring $k G$, the notation $\mathrm{Cell}^{G}(-)$ will stand for $\mathrm{Cell}_{k}^{k G}(-)$, so long as the ground $S$-algebra $k$ is understood. Note that a map $f: X \rightarrow Y$ of $G$-spaces which yields an isomorphism on all homotopy groups will induce a weak equivalence $\mathrm{C}_{*}(f ; k): \mathrm{C}_{*}(X ; k) \rightarrow \mathrm{C}_{*}(Y ; k)$ of $k G$-modules.

The classifying space of a topological group $G$ is denoted in the usual manner by B $G$ and $\mathrm{W} G$ is a contractible free $G$-space, such as the space described by Rothenberg and Steenrod [14].

Acknowledgements I am grateful to Professor Emmanuel Dror Farjoun for his guidance, support and for many useful suggestions. Most notably, it was he who led me to consider the Eilenberg-Moore spectral sequence in the context of cellular approximations. Part of this work was done while visiting the Centre De Recerca Mátematica in Barcelona under a Marie Curie scholarship. I would like to thank Professor Carles Casacuberta who enabled this visit.

\section{Cellular approximations}

Throughout this section we fix an $\mathbb{S}$-algebra $R$ and an $R$-module $k$. In this section we give the necessary background on cellular approximations and some of their basic properties. After defining $k$-cellularity and $k$ cellular-approximations, we recall $\mathrm{f}$ rom [7, Definition 4.6] the definition of proxy-smallness. By imposing the condition of proxy-smallness on $k$ one gets better handle on $k$-cellular approximations, as shown by Dwyer, Greenlees and Iyengar [7, Theorem 4.10]. We end this section with several examples in which $k$-cellularity has a simple description.

We recall the following definitions from [7].

Definition 2.1 A map $U \rightarrow V$ of $R$-modules is called a $k$-equivalence if the induced map of $\mathbb{S}$-modules $\operatorname{Hom}_{R}(k, U) \rightarrow \operatorname{Hom}_{R}(k, V)$ is an equivalence. An $R$-module $N$ 
is called $k-$ null if $\operatorname{Hom}_{R}(k, N) \simeq 0$. An $R$-module $M$ is called $k$-cellular if, for every $k$-null module $N, \operatorname{Hom}_{R}(M, N) \simeq 0$. Equivalently, $M$ is $k$-cellular if for every $k$-equivalence $f: U \rightarrow V$ the induced map $\operatorname{Hom}_{R}(M, U) \rightarrow \operatorname{Hom}_{R}(M, V)$ is an equivalence.

If $C \rightarrow X$ is a $k$-equivalence and $C$ is $k$-cellular then $C$ is called a $k$-cellular approximation of $X$ (or a $k$-cellularization of $X$ ). The module $C$ is denoted by $\operatorname{Cell}_{k}^{R}(X)$ or simply $\operatorname{Cell}_{k}(X)$ if $R$ is clear from the context.

Remark 2.2 Note that the property of being a $k$-cellular approximation can be defined solely in the derived category $\mathbf{D}_{R}$. Also, from Definition 2.1 it is easy to see that a $k$-cellular approximation of any given module is unique up to a unique isomorphism in the derived category $\mathbf{D}_{R}$.

An important example of cellular approximation was given by Dwyer and Greenlees in [6]. Suppose $R$ is a Noetherian commutative ring, let $I$ be an ideal of $R$ and take $k$ to be the ring $R / I$. Dwyer and Greenlees showed that for any $R$-module $X$ the homology groups of $\operatorname{Cell}_{k} X$ are isomorphic to the local cohomology of $X$ at $I$, ie:

$$
\pi_{-n}\left(\operatorname{Cell}_{k} X\right) \cong H_{I}^{n}(X)=\pi_{-n}\left(\operatorname{colim}_{m} \operatorname{Hom}\left(R / I^{m}, X\right)\right)
$$

Here is a simpler example. Suppose $R$ is a ring (not necessarily commutative) and $I$ is an ideal of $R$. Recall that a discrete $R$-module $M$ is said to be $I$-nilpotent if there is a filtration $0=M_{0} \subset M_{1} \subset \cdots \subset M_{n}=M$ such that $M_{i} / M_{i+1}$ is an $R / I$-module. It is a simple matter to show that every $I$-nilpotent module is $R / I$-cellular.

There is a well known equivalent definition of $k$-cellularity, which we will use extensively. To state this definition we must first recall what are thick and localizing subcategories.

Definition 2.3 Let $\mathcal{C}$ be a full subcategory of the category of $R$-modules. The subcategory $\mathcal{C}$ is called thick if it is closed under equivalences, triangles and retracts (direct summands). Closure under triangles means that given a triangle $X \rightarrow Y \rightarrow Z$ where two of the modules belong to $\mathcal{C}$, then so does the third. In particular a thick subcategory is closed under suspensions and finite coproducts. A thick subcategory is called localizing if in addition it is closed under arbitrary coproducts.

Let $A$ be an $R$-module. The smallest thick subcategory containing $A$ is called the thick subcategory generated by $A$. The localizing subcategory generated by $A$ is similarly defined. Following [7], we say an $R$-module $B$ is finitely built by $A$ if $B$ belongs to the thick subcategory generated by $A$. An $R$-module which is finitely built by $R$ is also called a small $R$-module. Finally, as in [7], we say $B$ is built by $A$ if $B$ 
belongs to the localizing subcategory generated by $A$. Clearly, if $B$ is finitely built from $A$ then $B$ is also built from $A$.

We can now state the equivalent definition for $k$-cellular modules: an $R$-module is $k$-cellular if and only if it is built from $k$. One part of this equivalence is easy to see, namely that any $R$-module built from $k$ is $k$-cellular. Using the techniques of [3] or [12, 5.1.5], one can show that for any $R$-module $X$ there exists a $k$-cellular approximation. This fact proves the other direction.

Remark 2.4 Let $S=\left\{A_{i}\right\}_{i \in I}$ be a set of $R$-modules. Say an $R$-module $M$ is built by $S$ (equivalently $S$-cellular) if $M$ belongs to the localizing subcategory generated by $S$. Clearly, this subcategory is equivalent to the localizing subcategory generated by the $R$-module $A=\bigoplus_{i \in I} A_{i}$. Therefore being $A$-cellular is the same as being $S$-cellular.

As noted above, given $R$-modules $k$ and $X$ one can always construct a $k$-cellular approximation for $X$. In general these constructions involve transfinite induction and a small object argument. To get a more manageable construction of $k$-cellular approximation, it is necessary to impose conditions on $k$. One such condition, given in [7, Definition 4.6] is that $k$ be proxy-small. We recall the definition of proxysmallness in Definition 2.5 below. Dwyer, Greenlees and Iyengar proved in [7, Theorem 4.10 ] that when $k$ is a proxy-small $R$-module the $k$-cellular approximation of any $R$-module $X$ is given by the natural map:

$$
\operatorname{Hom}_{R}(k, X) \otimes_{\operatorname{End}_{R}(k)} k \rightarrow X
$$

Definition 2.5 Call an $R$-module $k$ proxy-small if there exists an $R$-module $K$ such that $K$ is finitely built from $R, K$ is also finitely built from $k$ and $K$ builds $k$. The module $K$ is called a Koszul complex associated to $k$. (This is the term used in [7]; note that in [8] the module $K$ is called $a$ witness that $k$ is proxy-small.)

Remark 2.6 Suppose $k$ is proxy-small with an associated Koszul complex $K$. Because $K$ and $k$ build each other, an $R$-module $M$ is built from $k$ if and only if $M$ is built from $K$. Moreover, a map of $R$-modules is a $K$-equivalence if and only if it is a $k$-equivalence. Therefore a $K$-cellular approximation is the same as a $k$-cellular approximation.

In [7, Example 5.9], Dwyer, Greenlees and Iyengar show that for any finite group $G$ and any commutative ring $k$, the trivial $k G$ module $k$ is proxy-small. In Proposition 4.7 
below we will see that if $G$ is a finite nilpotent group and $k$ is a commutative $\mathbb{S}$-algebra such that $\pi_{0}(k)$ is an $\mathbb{F}_{p}$-algebra, then $k$ is a proxy-small $k G$-module.

We end this section by showing two cases where $k$-cellularity is relatively simple. In both cases $k$ is an $\mathbb{S}$-algebra which is given an $R$-module structure via a map of $\mathbb{S}$-algebras $R \rightarrow k$.

In the first case $R$ is a connective $\mathbb{S}$-algebra, ie $\pi_{n}(R)=0$ for all $n<0$. Recall that for an abelian group $A$, the appropriate Eilenberg-Mac Lane $\mathbb{S}$-module is denoted $H A$. By [10, Proposition IV.3.1], there exists map of $\mathbb{S}$-algebras $R \rightarrow H \pi_{0}(R)$ which yields the obvious isomorphism on the $\pi_{0}$-level. In particular, $H \pi_{0}(R)$ is an $R$-module. We show that when $R$ is connective, every bounded above $R$-module is $H \pi_{0}(R)$-cellular. This result is dual to a result of Bousfield from [1, Lemma 3.3f] over $\mathbb{S}$.

Proposition 2.7 Let $R$ be a connective $\mathbb{S}$-algebra, then every bounded above $R$ module is $H \pi_{0}(R)$-cellular.

Proof Let us denote the $\mathbb{S}$-algebra $H \pi_{0}(R)$ by $k$. Note that for any $R$-module $M$, the modules $H \pi_{i}(M)$ are $k$-modules and hence also $R$-modules. Let $X$ be a bounded above $R$-module. Connectivity of $R$ implies the existence of Postnikov sections in the category of $R$-modules (see [7, Lemma 3.2]). This allows us to use an argument of Dwyer and Greenlees from [6, Proposition 5.2], showing that $X$ is built from the $R$-modules $\left\{H \pi_{n}(X)\right\}_{n \in \mathbb{Z}}$. We detail this argument below.

Following [6], we denote by $M\langle-\infty, j\rangle$ the $j$-th Postnikov section of an $R$-module $M$. Thus, there is a natural map of $R$-modules $p_{j}: M \rightarrow M\langle-\infty, j\rangle$ such that $\pi_{k}\left(p_{j}\right)$ is an isomorphism for $k \leq j$ and $\pi_{m}(M\langle-\infty, j\rangle)$ is zero for $m>j$. Define $M\langle i, \infty\rangle$ to be the homotopy fiber of the map $M \rightarrow M\langle-\infty, i-1\rangle$, yielding a triangle:

$$
M\langle i, \infty\rangle \stackrel{c_{i}}{\rightarrow} M \stackrel{p_{i-1}}{\longrightarrow} M\langle-\infty, i-1\rangle
$$

Define $M\langle i, j\rangle$ (where $i \leq j$ ) to be

$$
(M\langle i, \infty\rangle)\langle-\infty, j\rangle
$$

(this notation agrees with the notation of the proof of [6, Proposition 5.2] for $R$-chain complexes, up to equivalence). Particularly useful is the triangle:

$$
M\langle i, j\rangle \rightarrow M\langle i-1, j\rangle \rightarrow M\langle i-1, i-1\rangle
$$

Also note that $M\langle i, i\rangle \simeq H \pi_{i}(M)$.

Here is the argument from [6, Proposition 5.2]. Since $X$ is bounded above, there is an index $j$ such that $\pi_{n}(X)=0$ for all $n>j$. First, it will be proved that for any $i \leq j$, 
the $R$-module $X\langle i, j\rangle$ is built from $\left\{H \pi_{n}(X)\right\}_{i \leq n \leq j}$. This statement is clearly true for $i=j$. It is true for $i<j$ by induction on $i$, using the triangle:

$$
X\langle i, j\rangle \rightarrow X\langle i-1, j\rangle \rightarrow X\langle i-1, i-1\rangle
$$

Second, there is an obvious equivalence $X\langle i, j\rangle \stackrel{\simeq}{\rightarrow} X\langle i, \infty\rangle$ and one can form a telescope

$$
X\langle j, j\rangle \rightarrow X\langle j-1, j\rangle \rightarrow \cdots \rightarrow X\langle i, j\rangle \rightarrow \cdots
$$

with obvious maps from this telescope to $X$. It is easy to see that the map

$$
\operatorname{hocolim}_{(i \rightarrow-\infty)} X\langle i, j\rangle \rightarrow X
$$

is an equivalence. Since $X\langle i, j\rangle$ is built by $\left\{H \pi_{n}(X)\right\}_{i \leq n \leq j}$, we conclude $X$ is built by $\left\{H \pi_{n}(X)\right\}_{n \leq j}$.

To finish the proof, note that for any $n, H \pi_{n}(X)$ is a $k$-module and therefore it is clearly built by $k$ in the category of $k$-modules. Hence $H \pi_{n}(X)$ is also built by $k$ in the category of $R$-modules.

Example 2.8 Let $k$ be a commutative ring (in the classical sense), let $G$ be a topological group and let $X$ be a $G$-space. Denote by $G_{0}$ the discrete group $\pi_{0}(G)$. It is easy to see that $\pi_{0}(k G) \cong k G_{0}$ and that the map of $\mathbb{S}$-algebras $k G \rightarrow k G_{0}$ is induced by the map of topological groups $G \rightarrow \pi_{0}(G)$. The group-ring $k G$ is connective, the $k G$-module $\mathrm{C}^{*}(X ; k)$ is coconnective, therefore, by Proposition 2.7 above, $\mathrm{C}^{*}(X ; k)$ is $k G_{0}$-cellular. In fact, $\mathrm{C}^{*}(X ; k)$ is built from the cohomology groups $H^{n}(X ; k)$ which are $k G_{0}$-cellular as $k G$-modules (because $H^{n}(X ; k)$ is a $k G_{0}$-module).

Example 2.9 Let $B$ be a finite connected nilpotent space with a fundamental group $G=\pi_{1}(B)$. A result of Hilton, Mislin and Roitberg [11, II.2.18] shows $G$ operates nilpotently on the modules $H_{*}(\widetilde{B})$, where $\widetilde{B}$ is the universal cover of $B$. Since $B$ is a finite space we see $C_{*}(\widetilde{B} ; \mathbb{Z})$ is a bounded above $\mathbb{Z} G$-module (ie a bounded above $\mathbb{Z} G$-chain complex $)$. From the proof of Proposition 2.7 we see that $C_{*}(\widetilde{B} ; \mathbb{Z})$ is built by it's homology groups. Every $H_{n}(\widetilde{B})$ is a nilpotent $\mathbb{Z} G$-module and hence $\mathbb{Z}$-cellular. Therefore $C_{*}(\widetilde{B} ; \mathbb{Z})$ is a $\mathbb{Z}$-cellular $\mathbb{Z} G$-chain complex.

This generalizes easily for any commutative ring $k$, by the following observation. It is a simple matter to show that applying the functor $k \otimes_{\mathbb{Z}}-$ turns $\mathbb{Z}$-cellular $\mathbb{Z} G$-modules into $k$-cellular $k G$-modules. Since $\mathrm{C}_{*}(\widetilde{B} ; k)$ is equivalent to $k \otimes_{\mathbb{Z}} \mathrm{C}_{*}(\widetilde{B} ; \mathbb{Z})$, it is a $k$-cellular $k G$-chain complex.

Moreover, this result can be generalized for any $\mathbb{S}$-algebra $k$ that is a commutative, connective and bounded above, by the following argument. Using the Atiyah-Hirzebruch 
spectral sequence (see eg [10, IV.3.7]) we see that $\pi_{n}\left(\mathrm{C}_{*}(\widetilde{B} ; k)\right)$ are nilpotent $\pi_{0}(k G)-$ modules. Since $k$ is bounded above, $\mathrm{C}_{*}(\widetilde{B} ; k)$ is also bounded above. Therefore $\mathrm{C}_{*}(\widetilde{B} ; k)$ is built from the nilpotent $\pi_{0}(k G)$-modules $H \pi_{n}\left(\mathrm{C}_{*}(\widetilde{B} ; k)\right)$. Since the modules $\pi_{n}\left(C_{*}(\widetilde{B} ; k)\right)$ are nilpotent, they are built by $\pi_{0}(k)$ in the category of $\pi_{0}(k G)$-modules. This implies $H \pi_{n}\left(\mathrm{C}_{*}(\widetilde{B} ; k)\right)$ is built by $H \pi_{0}(k)$ in the category of $k G$-modules. Since $H \pi_{0}(k)$ is a $k$-module, it is built by $k$ (also over $k G$ ). Therefore $\mathrm{C}_{*}(\widetilde{B} ; k)$ is a $k$-cellular $k G$-module.

Example 2.10 Here is a simple example of a connective $\mathbb{S}$-algebra $R$ and an unbounded $R$-module $M$ which is not $H \pi_{0}(R)$-cellular. Let $k=H \pi_{0}(R)$ and suppose $R$ is such that $\pi_{*}(R)$ is isomorphic to the graded ring $k_{*}[x]$, with $x$ in dimension greater than 0 . Let $X$ be the homotopy colimit of the telescope

$$
R \stackrel{x}{\rightarrow} \Sigma^{-|x|} R \stackrel{x}{\rightarrow} \Sigma^{-2|x|} R \stackrel{x}{\rightarrow} \cdots
$$

where $x: R \rightarrow \Sigma^{-|x|} R$ is a map representing multiplication by $x$. Clearly, $X$ is not bounded above. It is easy to show that $X$ is $k$-null and in particular $X$ is not $k$-cellular.

We now turn to the second case where $k$-cellularity is particularly simple. Here we suppose $k$ is a retract of $R$ in the derived category $\mathbf{D}_{R}$ and we obtain the following simple result, which will be put to good use in Section 4 .

Lemma 2.11 Suppose $k$ is an $R$-module via an $\mathbb{S}$-algebra map $a: R \rightarrow k$ and suppose there exists in $\mathbf{D}_{R}$ a map $b: k \rightarrow R$ such that $a b=\mathrm{id}_{k}$ (in $\mathbf{D}_{R}$ ). Then, for any $R$-module $X$, the $k$-cellular approximation of $X$ is given by the natural map:

$$
\operatorname{Hom}_{R}(k, X) \stackrel{a^{*}}{\rightarrow} X
$$

Remark 2.12 Recall that $\operatorname{Hom}_{R}(k, X)$ is taken in the derived sense, ie we take a cofibrant replacement of $k$ before applying the functor $\operatorname{Hom}_{R}(-, X)$. But now we must explain why this construction results in an $R$-module. The point is that we can replace $k$ by an $R$-bimodule which is cofibrant as a left $R$-module. So the remaining right action on this bimodule gives the desired left $R$-module structure on the function complex $\operatorname{Hom}_{R}(k, X)$.

Proof We start by showing the map $a \otimes 1: k \rightarrow k \otimes_{R} k$ is an equivalence. Clearly $(a \otimes 1)(b \otimes 1)=\mathrm{id}_{k}$ in $\mathbf{D}_{R}$ ie $b \otimes 1$ is a right inverse (in $\mathbf{D}_{R}$ ) for $a \otimes 1$. We need only show that $a \otimes 1$ has a left inverse in $\mathbf{D}_{R}$, since this would imply $b \otimes 1$ is both a left and right inverse for $a \otimes 1$. 
The map $a$ induces a functor $a^{*}: \mathbf{D}_{k} \rightarrow \mathbf{D}_{R}$ which is right adjoint to the functor $a_{*}=k \otimes_{R}$-. If $A$ is an $R$-module and $B$ is a $k$-module then the adjoint of a map $f: k \otimes_{R} A \rightarrow B$ is the composition $\left(a^{*} f\right) \circ\left(a \otimes_{R} 1\right): A \rightarrow a^{*} B$. Let $\mu: k \otimes_{R} k \rightarrow k$ be the map of $k$-modules that is adjoint to the identity map id $k$ via the adjunction above. Set $m=a^{*} \mu$, then the composition

$$
k \stackrel{a \otimes 1}{\longrightarrow} k \otimes_{R} k \stackrel{m}{\rightarrow} k
$$

is the map adjoint to $\mu$, hence it is the identity. This proves $m$ is a left inverse for $a \otimes 1$ in $\mathbf{D}_{R}$.

Given an $R$-module $X$ consider the map $a_{X}^{*}: \operatorname{Hom}_{R}(k, X) \rightarrow X$. Since $\operatorname{Hom}_{R}(k, X)$ is a $k$-module, it is $k$-cellular. All that is left is to show $a_{X}^{*}$ is a $k$-equivalence. Applying the functor $\operatorname{Hom}_{R}(k,-)$ to the map $a_{X}^{*}$ gives

$$
\operatorname{Hom}_{R}\left(k, a_{X}^{*}\right): \operatorname{Hom}_{R}\left(k, \operatorname{Hom}_{R}(k, X)\right) \rightarrow \operatorname{Hom}_{R}(k, X)
$$

which is equivalent to the map:

$$
\operatorname{Hom}_{R}\left(k \otimes_{R} k, X\right) \stackrel{(a \otimes 1)^{*}}{\longrightarrow} \operatorname{Hom}_{R}(k, X)
$$

Since $a \otimes 1$ is an equivalence, so is $(a \otimes 1)^{*}=\operatorname{Hom}_{R}(k, a \otimes 1)$.

\section{Change of rings}

Suppose we are given a map of $\mathbb{S}$-algebras $R \rightarrow S$ and an $S$-module $k$. Given another $S$-module $X$ there are two possible $k$-cellular approximations of $X$ we can consider: the $k$-cellular approximation over $S$, which is $\operatorname{Cell}_{k}^{S}(X)$ and the $k$-cellular approximation over $R$, which is $\operatorname{Cell}_{k}^{R}(X)$. We open this section by examining the relation between these two cellular approximations in the next lemma.

Lemma 3.1 Let $R \rightarrow S$ be a map of $\mathbb{S}$-algebras.

(1) (Strong independence of base) Let $k$ be an $S$-module. If $S \otimes_{R} k$ is $k$-cellular as an $S$-module, then for any $S$-module $X$ the map $\operatorname{Cell}_{k}^{S} X \rightarrow X$ is a $k$ cellular approximation of $X$ as an $R$-module. In particular $\operatorname{Cell}_{k}^{R} X \simeq \operatorname{Cell}_{k}^{S} X$ as $R$-modules.

(2) (Weak independence of base) Let $k$ be an $R$-module. If $S \otimes_{R} k$ is $k$-cellular as an $R$-module, then for any $S$-module $X$ the map $\operatorname{Cell}_{S \otimes_{R} k}^{S} X \rightarrow X$ is a $k$-cellular approximation of $X$ as an $R$-module. In particular $\operatorname{Cell}_{k}^{R} X \simeq$ Cell ${ }_{S \otimes_{R} k}^{S} X$ as $R$-modules. 
Remark 3.2 We have mentioned before the relation between cellular approximation and local cohomology of commutative Noetherian rings, which was proved in [6]. The independence of base property for local cohomology of commutative Noetherian rings is the following: Let $R \rightarrow S$ be a map of commutative Noetherian rings and let $I$ be an ideal of $R$. Then for every $S$-module $M$, the $I$-local cohomology groups of $M$, as an $R$-module, are isomorphic to the $I S$-local cohomology of groups $M$ as an $S$-module, where $I S$ is the ideal of $S$ generated by $I$.

Using Dwyer and Greenlees' result [6, Proposition 6.10] one can restate this property in cellular approximation terms. Indeed, it is easy to show that this independence of base property is nothing other than an equivalence of the cellular approximations: $\mathrm{Cell}_{R / I}^{R} M \simeq \mathrm{Cell}_{S / I S}^{S} M$ as $R$-modules.

We have dubbed the two parts of Lemma 3.1 as strong and weak independence of base, because they describe properties are analogous to the independence of base property for local cohomology. In fact, independence of base for local cohomology can be proved using the weak independence of base for cellular approximation and Dwyer and Greenlees' result.

Proof of Lemma 3.1 We begin by making the following observation. Suppose, as above, that $R \rightarrow S$ is a map of $\mathbb{S}$-algebras and $k$ is an $S$-module. Then every $S$-module that is $k$-cellular over $S$ is also $k$-cellular over $R$. This follows easily from the fact that the localizing subcategory of $S$-modules generated by $k$ is contained in the localizing subcategory of $R$-modules generated by $k$.

We turn to prove the strong independence of base property. Suppose $S \otimes_{R} k$ is $k-$ cellular as an $S$-module. Since $\operatorname{Cell}_{k}^{S} X$ is $k$-cellular as an $S$-module, it is also $k$-cellular as an $R$-module. We need only show that the map $\mu_{X}$ : $\operatorname{Cell}_{k}^{S} X \rightarrow X$ is a $k$-equivalence of $R$-modules. Using adjunctions between the $\otimes$ and Hom functors (see [10, Proposition III.6.3]) we get:

$$
\operatorname{Hom}_{R}\left(k, \mu_{X}\right) \simeq \operatorname{Hom}_{R}\left(k, \operatorname{Hom}_{S}\left(S, \mu_{X}\right)\right) \simeq \operatorname{Hom}_{S}\left(S \otimes_{R} k, \mu_{X}\right)
$$

The map $\mu_{X}$ is a $k$-equivalence of $S$-modules. The $S$-module $S \otimes_{R} k$ is $k$-cellular and therefore $\mu_{X}$ is also an $S \otimes_{R} k$-equivalence. This proves $\operatorname{Hom}_{R}\left(k, \mu_{X}\right)$ is an equivalence.

We now prove the weak independence of base property. Let $k$ be an $R$-module and suppose $S \otimes_{R} k$ is $k$-cellular as an $R$-module. As above, $\operatorname{Cell}_{S \otimes_{R} k}^{S} X$ is built by $S \otimes_{R} k$ also over $R$. Since $S \otimes_{R} k$ is built by $k$ over $R$, we see Cell ${ }_{S \otimes_{R} k} X$ is also built by $k$ over $R$. We are left with showing the map $v_{X}$ : Cell ${ }_{S \otimes_{R} k}^{S} X \rightarrow X$ is a 
$k$-equivalence of $R$-modules. Using the same adjunctions as before, we get:

$$
\operatorname{Hom}_{R}\left(k, v_{X}\right) \simeq \operatorname{Hom}_{S}\left(S \otimes_{R} k, v_{X}\right)
$$

Since $\operatorname{Hom}_{S}\left(S \otimes_{R} k, v_{X}\right)$ is an equivalence, we see that $v_{X}$ is a $k$-equivalence of $R$-modules.

The main application of Lemma 3.1 above is to group-rings. We start with discrete groups. Fix a commutative $\mathbb{S}$-algebra $k$ and a short exact sequence of discrete groups: $N \rightarrow G \rightarrow Q$. The augmentation map of $\mathbb{S}$-algebras $k G \rightarrow k$ makes $k$ into a $k G$-module. We denote $k$-cellular approximation over the group-ring $k G$ by $\mathrm{Cell}^{G}$ whenever $k$ is clear from the context. In similar fashion, we will refer to $k G$-modules as $G$-modules whenever $k$ is clear from the context. We say Cell ${ }^{G}$ is trivial if for every $k G$-module $X$ the map $\operatorname{Cell}^{G} X \rightarrow X$ is an equivalence.

The maps of groups induce maps $k N \rightarrow k G \rightarrow k Q \rightarrow k$ of $\mathbb{S}$-algebras. For any $G$-module $X$ there are two possible $k$-cellular approximations: $\mathrm{Cell}^{G} X$ and $\mathrm{Cell}^{N} X$, each over a different ring. Similarly, for a $Q$-module $Y$ we can consider two $k$-cellular approximations: $\mathrm{Cell}^{Q} Y$ and $\mathrm{Cell}^{G} Y$. The relations between the various possible $k$-cellular approximations are given in the next proposition.

Proposition 3.3 Let $N \rightarrow G \rightarrow Q$ be a short exact sequence of discrete groups and let $k$ be a commutative $\mathbb{S}$-algebra.

(1) For every $G$-module $X$ the map $\operatorname{Cell}_{k Q}^{G} X \rightarrow X$ is a $k$-cellular approximation of $X$ as an $N$-module. In particular there is an weak equivalence of $N$-modules: $\mathrm{Cell}^{N} X \simeq \operatorname{Cell}_{k Q}^{G} X$.

(2) If Cell ${ }^{Q}$ is trivial then for every $G$-module $X$ the map $\mathrm{Cell}^{G} X \rightarrow X$ is a $k$-cellular approximation of $X$ as an $N$-module. In particular there is an equivalence of $N$-modules: $\mathrm{Cell}^{G} X \simeq \mathrm{Cell}^{N} X$.

(3) If both $\mathrm{Cell}^{Q}$ and $\mathrm{Cell}^{N}$ are trivial, then $\mathrm{Cell}^{G}$ is also trivial.

(4) If $\mathrm{C}_{*}(\mathrm{~B} N ; k)$ is a $k$-cellular $Q$-module, then for any $Q$-module $Y$ the map $\mathrm{Cell}^{Q} Y \rightarrow Y$ is a $k$-cellular approximation of $Y$ as a $G$-module. In particular there is an equivalence of $G$-modules: $\mathrm{Cell}^{Q} Y \simeq \mathrm{Cell}^{G} Y$.

Before proving this proposition we need the following lemma.

Lemma 3.4 The module $k G \otimes_{k N} k$ is $k$-cellular as an $N$-module.

Proof Clearly $G$ is isomorphic to $\bigsqcup_{q \in Q} N$ as an $N$-set. Hence $K G \simeq \bigoplus_{q \in Q} k N$ as a $k N$-module. The result follows. 
Proof of Proposition 3.3 The previous lemma implies that for the map $k N \rightarrow k G$ and the $G$-module $k$ the weak independence of base property holds (Lemma 3.1). This proves the first item on our list.

To prove the second item we suppose Cell ${ }^{Q}$ is trivial. This implies $k Q$ is $k$-cellular as a $Q$-module. Since $k$ is clearly $Q$-cellular as a $Q$-module, we see $k$ and $k Q$ build each other over $k Q$. But then $k$ and $k Q$ build each other also over $k G$. We see that for every $G$-module $X$ the map $\operatorname{Cell}_{k Q}^{G} X \rightarrow X$ is a $k$-cellular approximation of $X$ as a $G$-module. To finish the proof of the second part of the proposition we apply the first part, which was proved above.

Now suppose both Cell $Q$ and Cell ${ }^{N}$ are trivial and let $X$ be a $G$-module. Because $\mathrm{Cell}^{Q}$ is trivial, the natural map $\mathrm{Cell}^{G} X \rightarrow X$ of $G$-modules is a $k$-cellular approximation of $X$ as an $N$-module. Since Cell ${ }^{N}$ is trivial, this map is an equivalence of $N$-modules, hence it is also an equivalence of $G$-modules. This proves the third item on our list.

For the last item note that $\mathrm{C}_{*}(\mathrm{~B} N ; k) \simeq k Q \otimes_{G} k$. Thus the result follows from strong independence of base (Lemma 3.1).

Example 3.5 Let $N \rightarrow G \rightarrow Q$ be a short exact sequence of finite groups with $N$ being a central subgroup of $G$ and $Q$ an abelian group. Let $k$ be a commutative ring. It is easy to show that the action of $Q$ on $H_{*}(\mathrm{~B} N ; k)$ is trivial, ie every element of $Q$ acts as the identity. Since $Q$ is abelian, the results of Dwyer and Greenlees [6, Propositions 5.3 and 6.9] show that $\mathrm{C}_{*}(\mathrm{~B} N ; k)$ is a $k$-cellular $k Q$-module. So, by Proposition 3.3 for every $k Q$-module $M$, the map Cell ${ }^{Q} M \rightarrow M$ is a $k$-cellular approximation of $M$ as a $k G-$ module.

Example 3.6 Here is an example where there is no independence of base property. Let $\Sigma_{3}$ be the symmetric group on three elements. There is a short exact sequence of groups

$$
C_{3} \rightarrow \Sigma_{3} \rightarrow C_{2}
$$

where $C_{n}$ is the cyclic group of $n$ elements. Let $R$ be the group ring $\mathbb{Z} \Sigma_{3}$, let $S$ be the group-ring $\mathbb{Z} C_{2}$ and let $k$ be the $S$-module $\mathbb{Z}$ with trivial $C_{2}$-action. As noted in the previous example, $S \otimes_{R} k$ is equivalent to the chains of the classifying space of $C_{3}$, namely $\mathrm{C}_{*}\left(\mathrm{~B} C_{3} ; \mathbb{Z}\right)$. Recall $H_{1}\left(\mathrm{~B} C_{3} ; \mathbb{Z}\right) \cong \mathbb{Z} / 3$ (the group $H_{1}\left(\mathrm{~B} C_{3} ; \mathbb{Z}\right.$ ) is $\pi_{1}\left(\mathrm{C}_{*}\left(\mathrm{~B} C_{3} ; \mathbb{Z}\right)\right)$ in our usual notation). It is easy to see that the $C_{2}$ action on $H_{1}\left(\mathrm{BC} C_{3} ; \mathbb{Z}\right)$ is simply exchanging the two generators of $\mathbb{Z} / 3$.

Let $I$ be the augmentation ideal of $S$, ie the kernel of the map $\mathbb{Z} C_{2} \rightarrow \mathbb{Z}$. Had $S \otimes_{R} k$ been $k$-cellular as an $S$-module, then $H_{1}\left(\mathrm{~B} C_{3} ; \mathbb{Z}\right)$ would have been a nilpotent $S-$ module by [6, Proposition 6.11]. But it is easy to see that $H_{1}\left(\mathrm{~B} C_{3} ; \mathbb{Z}\right)$ is not a nilpotent 
$S$-module. Moreover, this argument also shows that $S \otimes_{R} k$ is not $k$-cellular even as an $R$-module.

We turn to discuss the applications of Lemma 3.1 to topological groups. We start with the following example.

Example 3.7 Let $B$ be a finite connected nilpotent space and $k$ a commutative ring. The map $\Omega B \rightarrow \pi_{1}(B)$ of topological groups induces a map $k[\Omega B] \rightarrow k\left[\pi_{1}(B)\right]$, we show this map has the strong independence of base property.

Let $\widetilde{B}$ be the universal cover of $B$. The following is a homotopy fibration sequence:

$$
\widetilde{B} \rightarrow B \rightarrow K\left(\pi_{1}(B), 1\right)
$$

The map of $\mathbb{S}$-algebras we have in mind is the induced map of group rings: $k[\Omega B] \rightarrow$ $k\left[\pi_{1}(B)\right]$. As noted in Example 2.9, $\mathrm{C}_{*}(\widetilde{B} ; k)$ is a $k$-cellular $k\left[\pi_{1}(B)\right]$-module. Note that $k\left[\pi_{1}(B)\right] \otimes_{k[\Omega B]} k$ is equivalent to $\mathrm{C}_{*}(\widetilde{B} ; k)$. This implies strong independence of base (Lemma 3.1), ie for any $k\left[\pi_{1}(B)\right]$-module $X$ the map Cell ${ }^{\pi_{1}(B)} X \rightarrow X$ is a $k$-cellular approximation of $X$ as a $k[\Omega B]$-module (strong independence of base). The importance of this property will become clear in Section 5 .

The general property alluded to in Example 3.7 is given in the next proposition. Note that if $F \rightarrow E \rightarrow B$ is a homotopy fibration sequence of spaces with $B$ a connected space, then $F$ is equivalent to a $\Omega B$-space (we recall this standard construction at the beginning of Section 5$)$. Hence we assume that $\mathrm{C}_{*}(F ; k)$ is a $k[\Omega B]$-space.

Proposition 3.8 Let $F \rightarrow E \rightarrow B$ be a homotopy fibration sequence of spaces with $B$ and $E$ connected and let $k$ be a commutative $\mathbb{S}$-algebra. Suppose $\mathrm{C}_{*}(F ; k)$ is a $k-$ cellular $k[\Omega B]$-module. Then for every $k[\Omega B]$-module $X$ the map Cell ${ }^{k[\Omega B]} X \rightarrow X$ is a $k$-cellular approximation of $X$ as a $k[\Omega E]$-module.

Proof From Lemma 3.1 we see it is enough to show that $k[\Omega B] \otimes_{k[\Omega E]} k$ is a $k$-cellular $k[\Omega B]$-module. Hence it would suffice to show that $k[\Omega B] \otimes_{k[\Omega E]} k$ is equivalent to $\mathrm{C}_{*}(F ; k)$ as $k[\Omega B]-$ modules.

Consider the homotopy fibration sequence $\Omega B \rightarrow F \rightarrow E$. The Borel construction gives an equivalence $\Omega B \times \Omega E \mathrm{~W} \Omega E \simeq F$. Moreover, the map giving this equivalence is map of $\Omega B$-spaces. Thus we have an equivalence of $k[\Omega B]$-modules:

$$
\mathrm{C}_{*}\left(\Omega B \times{ }_{\Omega E} \mathrm{~W} \Omega E ; k\right) \simeq \mathrm{C}_{*}(F ; k)
$$


To finish the proof we use the results of Elmendorf, Kriz, Mandell and May [10, Proposition IV.7.5 and Theorem IV.7.8], which show that:

$$
\mathrm{C}_{*}\left(\Omega B \times_{\Omega E} \mathrm{~W} \Omega E ; k\right) \simeq \mathrm{C}_{*}(\Omega B ; k) \otimes_{k[\Omega E]} \mathrm{C}_{*}(\mathrm{~W} \Omega E ; k)
$$

Since $\mathrm{C}_{*}(\mathrm{~W} \Omega E ; k) \simeq k$, we are done.

Example 3.9 Let $G$ be a discrete finite group. Take an embedding of $G$ into the group $\mathrm{SU}(n)$, there is always such an embedding for a large enough $n$. This embedding makes $\mathrm{SU}(n)$ into a $G$-space. Fix a commutative ring $k$. We show that the principal fibration sequence

$$
\mathrm{SU}(n) \rightarrow \mathrm{SU}(n) / G \rightarrow \mathrm{B} G
$$

and the commutative ring $k$ satisfy the conditions of Proposition 3.8. All we need is to show $\mathrm{C}_{*}(\mathrm{~B} \Omega \mathrm{SU}(n) ; k)$ is a $k$-cellular $G$-module. It is easy to see that the $G$-action on $\mathrm{B} \Omega \mathrm{SU}(n) \simeq \mathrm{SU}(n)$ comes from the embedding of groups $G \rightarrow \mathrm{SU}(n)$. In [7, Example 5.9], Dwyer Greenlees and Iyengar show that this map makes $\mathrm{C}_{*}(\mathrm{SU}(n) ; k)$ into a $k$-cellular $k G$-module.

We end this section with an example of a topological group $G$ where the map $k G \rightarrow$ $k\left[\pi_{0}(G)\right]$ does not have strong independence of base property.

Example 3.10 Fix some natural number $n$ and consider the following fibration of pointed spaces:

$$
S^{n} \rightarrow \mathbb{R P}^{n} \rightarrow \mathrm{B} C_{2}
$$

As in Example 3.7 we set $R=\mathbb{Z}\left[\Omega \mathbb{R}^{n}\right]$ (recall this is $C_{*}\left(\Omega \mathbb{R} \mathbb{P}^{n} ; \mathbb{Z}\right)$ ) and $S=$ $\mathbb{Z}\left[\Omega \mathrm{B} C_{2}\right]$. The weak equivalence of topological groups $\Omega \mathrm{B} C_{2} \simeq C_{2}$ induces an equivalence between the derived category of $S$-modules and the derived category of $\mathbb{Z}\left[C_{2}\right]$-modules (see [10, Theorem III.4.2]). So we will work in the category of $\mathbb{Z}\left[C_{2}\right]$-modules instead of the category of $S$-modules.

The action of $C_{2}$ on the $n$-sphere $S^{n}$ is by the antipodal map. If $n$ is odd this map is homotopy equivalent to the identity. This implies that we are in the same situation as in Example 3.7. However, if $n$ is even, then the antipodal map induces a nontrivial action on the reduced homology groups of the $n$-sphere $S^{n}$. Thus $\pi_{n}\left(\mathrm{C}_{*}\left(S^{n} ; \mathbb{Z}\right)\right)$ is a nontrivial $C_{2}$-module, the $C_{2}$-action being multiplication by -1 . We will show this $C_{2}$-module, which we denote by $\widetilde{\mathbb{Z}}$, is not $\mathbb{Z}$-cellular as a $\mathbb{Z}\left[C_{2}\right]$-module (we consider $\mathbb{Z}$ to be a $\mathbb{Z}\left[C_{2}\right]$-module with the trivial action of $C_{2}$ ).

The ring $\mathbb{Z}\left[C_{2}\right]$ is commutative and its augmentation ideal is finitely generated, so by [6, Proposition 6.11] a finitely generated $\mathbb{Z}\left[C_{2}\right]$-chain complex is $\mathbb{Z}$-cellular if and only if all of its homology groups are nilpotent discrete $\mathbb{Z}\left[C_{2}\right]$-modules. It is easy to 
see that $\widetilde{\mathbb{Z}}$ is not a nilpotent module and therefore $\mathrm{C}_{*}\left(S^{n} ; \mathbb{Z}\right)$ described above is not $\mathbb{Z}$-cellular as a $\mathbb{Z}\left[C_{2}\right]$-module.

\section{Cellular approximation in nilpotent groups}

Fix a prime number $p$ and let $k$ be a commutative $\mathbb{S}$-algebra such that $\pi_{0}(k)$ is an $\mathbb{F}_{p}$-algebra. In this section we consider $k$-cellular approximation over a group-ring $k G$, where $G$ is a finite group. When $G$ is a $p$-group we show that every $k P$-module is $k$-cellular, this is done in Corollary 4.3. For a finite nilpotent group $G$ we give a nice formula for $k$-cellular approximation over $k G$ in Proposition 4.4. We further show, in Proposition 4.7, that $k$ is proxy-small as a $k G$-module.

\subsection{Finite $p$-groups}

Recall $\pi_{0}(k)$ is an $\mathbb{F}_{p}$-algebra. This implies the multiplication by $p$ map $\cdot p: k \rightarrow k$ is equal to the zero map in $\mathbf{D}_{k}$. Using this fact we will show that if $P$ is a finite $p$ group, then $k$ finitely builds $k P$. In particular we get that $k$ is a proxy-small $k P$-module and hence every $k P$-module is $k$-cellular. These results are obvious when $k$ is a commutative $\mathbb{F}_{p}$-algebra and the generalization to the case where $k$ is an $\mathbb{S}$-algebra is simple.

Proposition 4.1 Let $P$ be a finite $p$-group and $k$ a commutative $\mathbb{S}$-algebra such that $\pi_{0}(k)$ is an $\mathbb{F}_{p}$-algebra. Then $k$ finitely builds $k P$ in the category of $k P$-modules and therefore $k$ is a proxy-small $k P$-module.

We start by proving the following lemma.

Lemma 4.2 Let $C$ be a finite cyclic group with a generator $g$ and let $k$ be any commutative $\mathbb{S}$-algebra. There is a triangle

$$
\Sigma k \rightarrow K \rightarrow k
$$

where $K$ is the homotopy cofiber of the map $k C \stackrel{1-g}{\longrightarrow} k C$.

Note that the construction of such a triangle is obvious when $k$ is a commutative ring. Indeed there is a short exact sequence:

$$
0 \rightarrow k \rightarrow k C \stackrel{1-g}{\longrightarrow} k C \rightarrow k \rightarrow 0
$$


Proof of Lemma 4.2 Let us begin with the case where $k$ is the sphere spectrum $\mathbb{S}$. By [10, Proposition IV.3.1] there is a map of $\mathbb{S}$-algebras $\mathbb{S} C \rightarrow H \pi_{0}(\mathbb{S} C)$. Note that $H \pi_{0}(\mathbb{S} C) \cong H \mathbb{Z} C$. Clearly $H \mathbb{Z} C \otimes_{\mathbb{S} C} K$ is equivalent to the cone of the map $1-g: \mathbb{Z} C \rightarrow \mathbb{Z} C$ and hence:

$$
\pi_{i}\left(H \mathbb{Z} C \otimes_{\mathbb{S} C} K\right)= \begin{cases}\mathbb{Z} & i=0,1, \\ 0 & \text { otherwise }\end{cases}
$$

The augmentation map $\mathbb{S} C \rightarrow \mathbb{S}$ induces a map $f: K \rightarrow \mathbb{S}$. There is also a map $\mathbb{S} \rightarrow \mathbb{S} C$ of $\mathbb{S} C$-modules, which is defined by $1 \mapsto \sum_{x \in C} x$. One can easily check this is indeed a map of $\mathbb{S} C$-modules and it induces a map $g: \Sigma \mathbb{S} \rightarrow K$. Let $F$ be the homotopy fiber of the map $f$, then clearly:

$$
\pi_{i}\left(H \mathbb{Z} C \otimes_{\mathbb{S} C} F\right)= \begin{cases}\mathbb{Z} & i=1, \\ 0 & \text { otherwise }\end{cases}
$$

Since the composition $\mathbb{S} \rightarrow \mathbb{S} C \stackrel{1-g}{\longrightarrow} \mathbb{S} C$ is zero, one sees that the map $g$ induces a map $g^{\prime}: \Sigma \mathbb{S} \rightarrow F$. It is easy to check that the induced map

$$
H \mathbb{Z} C \otimes_{\mathbb{S} C} \Sigma \mathbb{S} \rightarrow H \mathbb{Z} C \otimes_{\mathbb{S} C} F
$$

is an equivalence. Consider the mapping cone $\operatorname{Cone}\left(g^{\prime}\right)$. This $\mathbb{S C}$-module is bounded below, simply because of it's construction. In addition, $H \mathbb{Z} C \otimes_{\mathbb{S} C} \operatorname{Cone}\left(g^{\prime}\right) \simeq 0$. Hence, by [10, IV.3.6], Cone $\left(g^{\prime}\right) \simeq 0$ and the map $g^{\prime}$ is an equivalence, yielding the desired triangle:

$$
\Sigma \mathbb{S} \rightarrow K \rightarrow \mathbb{S}
$$

Now suppose $k$ is any commutative $\mathbb{S}$-algebra. Note that: $k \simeq k \otimes_{\mathbb{S}} \mathbb{S}$ and $k C \simeq$ $k \otimes_{\mathbb{S}} \mathbb{S} C$. Hence applying the functor $k \otimes_{\mathbb{S}}-$ to the triangle $\Sigma \mathbb{S} \rightarrow K \rightarrow \mathbb{S}$ gives the triangle: $\Sigma k \rightarrow K \rightarrow k$.

Proof of Proposition 4.1 We start with the case where $P$ is the cyclic group of $p$ elements. Let $g$ be a generator of $P$ and let $K$ be the homotopy cofiber of the map $1-g: k P \rightarrow k P$. By the previous lemma, $k$ finitely builds $K$. Denote by $K_{n}$ the homotopy cofiber of the map $(1-g)^{n}: k P \rightarrow k P$. Consider the following commutative diagram whose rows and columns are triangles:

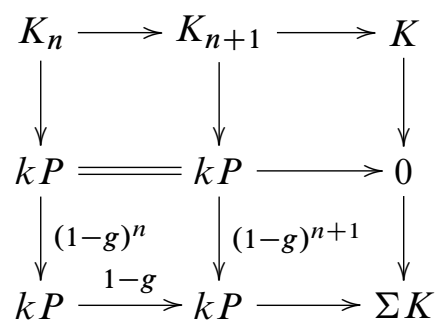


From the top row we see, by induction on $n$, that $K_{n}$ is finitely built from $K$. In particular $K_{p}$ is finitely built by $K$. Since the map $(1-g)^{p}$ is equivalent to the zero map, the module $K_{p}$ is equivalent to $k P \oplus \Sigma^{-1} k P$. Therefore $K_{p}$ finitely builds $k P$ and hence $k$ finitely builds $k P$. This completes the case where $P$ is the cyclic group with $p$-elements.

Now suppose $P$ is any finite $p$-group. We prove the proposition by induction on the order of $P$. Since $P$ is a finite $p$-group, there is a short exact sequence of groups

$$
N \hookrightarrow P \rightarrow C_{p}
$$

where $C_{p}$ is the cyclic group with $p$-elements. By the induction assumption, $k$ finitely builds $k N$ in the category of $k N$-modules. By applying the functor $k P \otimes_{k N}-$ to the recipe of constructing $k N$ from $k$, we see that $k P \otimes_{k N} k \simeq k C_{p}$ finitely builds $k P \otimes_{k N} k N \simeq k P$ in the category of $k P$-modules.

We saw that $k$ finitely builds $k C_{p}$ in the category of $k C_{p}$-modules. Hence $k$ also finitely builds $k C_{p}$ in the category of $k P$-modules. We conclude $k$ finitely builds $k P$ in the category of $k P$-modules.

Finally, $k P$ is a Koszul-complex for $k$, since $k P$ is a small $k P$-module, $k$ finitely builds $k P$, and clearly $k P$ builds $k$.

Here is the obvious corollary of Proposition 4.1.

Corollary 4.3 Let $P$ be a finite $p$-group and $k$ a commutative $\mathbb{S}$-algebra such that $\pi_{0}(k)$ is an $\mathbb{F}_{p}$-algebra. Then every $k P$-module is $k$-cellular.

\subsection{Finite nilpotent groups}

Let $N$ be a finite nilpotent group. As before, $p$ is a fixed prime number and $k$ is a commutative $\mathbb{S}$-algebra such that $\pi_{0}(k)$ is an $\mathbb{F}_{p}$-algebra. Let $P$ be a $p$-Sylow subgroup of $N$ and let $H=N / P$. Since $N$ is nilpotent, it is isomorphic to the group $P \times H$.

Proposition 4.4 Let $N, P, H$ and $k$ be as above. Then for any $N$-module $X$, the map $\operatorname{Hom}_{k N}(k P, X) \rightarrow X$ (induced by the map $k N \rightarrow k P$ ) is the $k$-cellular approximation of $X$ as an $N$-module.

Proof There is a short exact sequence of groups: $1 \rightarrow H \rightarrow N \rightarrow P \rightarrow 1$. As we saw in Proposition 4.1, $k$ builds $k P$. Hence $k$-cellular approximations are the same as $k P$-cellular approximations. 
Since $P$ is a $p$-Sylow subgroup, the order of $H$ is prime to $p$. This implies the map $\cdot|H|: k \rightarrow k$ (multiplication by the order of $H$ ) is an equivalence. Let $b: k \rightarrow k H$ be the map defined (up to homotopy) by sending 1 to $\Sigma_{h \in H} h$. The composition $k \rightarrow k H \rightarrow k$ is the multiplication by $|H|$ map, hence an equivalence. Thus the augmentation map $k H \rightarrow k$ has a right inverse, showing $k$ is a retract of $k H$ (in the derived category $\mathbf{D}_{k H}$ ). Applying the functor $k N \otimes_{k H}-$ to these maps yields two maps: $k P \rightarrow K N \rightarrow k P$ whose composition is the identity (in $\mathbf{D}_{k N}$ ). Note that the right map is the one induced by the map $N \rightarrow P$ of groups. Now we use Lemma 2.11, showing: $\operatorname{Hom}_{k N}(k P, X) \rightarrow X$ is a $k$-cellular approximation of $X$ as a $k H$-module.

Remark 4.5 Note that the map $\operatorname{Hom}_{k N}(k P, X) \rightarrow X$ is equivalent to the map $\operatorname{Hom}_{k H}(k, X) \rightarrow X$ by well known adjunctions. Suppose that $k=\mathbb{F}_{p}$, then $k$ is a projective $H$-module (because it is a retract of $k H$ ). This implies the derived functor $\operatorname{Hom}_{k H}(k,-)$ is equivalent to the nonderived version. So, for a $k N$-chain complex $X$, Cell $^{N} X$ is the fixed points of the $H$-action on $X$, commonly denoted $X^{H}$.

In fact, the proof of Proposition 4.4 implies a slightly more general result:

Proposition 4.6 Let $H \rightarrow G \rightarrow P$ be a short exact sequence of finite groups, with $P$ being a $p$-group. Suppose that the order of $H$ is prime to $p$ and that $\pi_{0}(k)$ is an $\mathbb{F}_{p}$-algebra, then for any $k G$-module $X$ the natural map $\operatorname{Hom}_{k G}(k P, X) \rightarrow X$ is a $k$-cellular approximation of $X$ as a $k G$-module.

We also show that $k$ is proxy-small as a $k N$-module. This will later enable us to use Dwyer, Greenlees and Iyengar's formula for cellular approximation [7, Theorem 4.10].

Proposition 4.7 Let $N$ be a finite nilpotent group and $k$ a commutative $\mathbb{S}$-algebra such that $\pi_{0}(k)$ is an $\mathbb{F}_{p}$-algebra. Then $k$ is a proxy-small $k N$-module.

Proof As before let $P$ be the $p$-Sylow subgroup of $N$ and let $H=N / P$ so $N \cong P \times H$. We will show that $k P$ is a Koszul complex for $k$ over $k N$. From the proof of Proposition 4.4 above we see that $k$ is a retract of $k H$ (in $\mathbf{D}_{k N}$ ). This implies $k N \otimes_{k H} k \simeq k P$ is a retract of $k N \otimes_{k H} k H \simeq k N$. Hence $k P$ is small as an $N$-module.

From Proposition 4.1 we see $k$ finitely builds $k P$ over $k P$ and therefore $k$ also finitely builds $k P$ over $k N$. Clearly $k P$ builds $k$ over $k N$, since $k$ is a $k P$-module. Hence $k P$ is a Koszul-complex for $k$ over $k N$. 


\section{Relation with the target of the Eilenberg-Moore spectral sequence}

Fix a fibration sequence $F \rightarrow E \rightarrow B$, where $E$ and $B$ are always assumed to be connected spaces. Let $\mathrm{W} \Omega B$ be a contractible space with a free $\Omega B$-action (such as the one described by Rothenberg and Steenrod in [14]). The pullback of the diagram

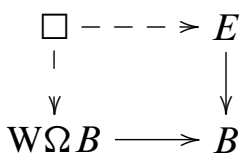

is a (right) $\Omega B$-space equivalent to $F$. So, without loss of generality, we will assume $F$ is a $\Omega B$-space. Let $k$ be a commutative $\mathbb{S}$-algebra. In this section the chains of spaces are always taken with coefficients in $k$, therefore we omit $k$ from the notation $\mathrm{C}_{*}(-; k)$. We use $R$ to denote the group-ring $k[\Omega B]$, ie $\mathrm{C}_{*}(\Omega B ; k)$. So $\mathrm{C}_{*}(F)$ and $\mathrm{C}^{*}(F)$ are both $R$-modules.

The results of Dwyer, Greenlees and Iyengar imply that, under some conditions on the space $B$, the natural map $\mathrm{C}^{*}(E) \otimes_{\mathrm{C}^{*}(B)} k \rightarrow \mathrm{C}^{*}(F)$ is a $k$-cellular approximation of $C^{*}(F)$ over $R$ (see [7, Theorem 4.10]). These conditions on $B$ are specified in Lemma 5.6 below. Applying the machinery we have set up in the previous section to the $k$-cellular approximation of $\mathrm{C}^{*}(F)$ over $R$, we obtain the following theorems.

Theorem 5.1 Fix a prime $p$. Let $N$ be a finite nilpotent group and let $P \subseteq N$ be the $p$-Sylow subgroup of $N$, so $N \cong P \times H$ with the order of $H$ being prime to $p$. Let $F \rightarrow E \rightarrow \mathrm{B} N$ be a homotopy fibration sequence over the classifying space of $N$, with $E$ being a connected space. If $k$ is any commutative $\mathbb{S}$-algebra such that $\pi_{0}(k)$ is an $\mathbb{F}_{p}$-algebra, then

$$
\mathrm{C}^{*}(E ; k) \otimes_{\mathrm{C}^{*}(B ; k)} k \simeq \mathrm{C}^{*}\left(F_{h(H)}\right)
$$

where $F_{h(H)}$ is the homotopy orbit space of $F$ with respect to the $H$-action on $F$. In particular, if $N=P$ then:

$$
\mathrm{C}^{*}(E ; k) \otimes_{\mathrm{C}^{*}(B ; k)} \simeq \simeq \mathrm{C}^{*}(F ; k)
$$

Theorem 5.2 Fix a prime $p$. Let $B$ be a finite connected nilpotent space with a finite fundamental group $N=\pi_{1}(B)$. Let $P \subseteq N$ be the $p$-Sylow subgroup of $N$, so $N \cong P \times H$. Let $F \rightarrow E \rightarrow B$ be a homotopy fibration sequence over $B$, where $E$ is a connected space. Then

$$
\operatorname{tor}_{-n}^{\mathrm{C}^{*}\left(B ; \mathbb{F}_{p}\right)}\left(\mathrm{C}^{*}\left(E ; \mathbb{F}_{p}\right), \mathbb{F}_{p}\right)=H^{n}\left(F ; \mathbb{F}_{p}\right)^{H}
$$

where $H^{n}\left(F ; \mathbb{F}_{p}\right)^{H}$ are the fixed points of the $H$-action on $H^{n}\left(F ; \mathbb{F}_{p}\right)$. 
Remark 5.3 The tensor product $\mathrm{C}^{*}(E) \otimes_{\mathrm{C}^{*}(B)} k$ needs, perhaps, some clarification. The maps $E \rightarrow B$ and $\mathrm{pt} \rightarrow B$ induce maps of $k$-algebras $\mathrm{C}^{*}(B) \rightarrow \mathrm{C}^{*}(E)$ and $\mathrm{C}^{*}(B) \rightarrow k$. These maps make $\mathrm{C}^{*}(E)$ and $k$ into $\mathrm{C}^{*}(B)$-bimodules. Considering $\mathrm{C}^{*}(E)$ as a right $\mathrm{C}^{*}(B)$-module and $k$ as a left $\mathrm{C}^{*}(B)$-module, we form the tensor product: $\mathrm{C}^{*}(E) \otimes_{\mathrm{C}^{*}(B)} k$.

Remark 5.4 The Eilenberg-Moore spectral sequence is of the form:

$$
E_{p, q}^{2}=\operatorname{tor}_{p, q}^{\pi_{*}^{*}\left(\mathrm{C}^{*}(B)\right)}\left(\pi_{*}\left(\mathrm{C}^{*}(E)\right), \pi_{*}(k)\right) \Rightarrow \pi_{p+q}\left(\mathrm{C}^{*}(E) \otimes_{\mathrm{C}^{*}(B)} k\right)
$$

The version of the Eilenberg-Moore spectral sequence we consider is the one given in [10, Theorem IV.4.1]. Its convergence properties are also described there and elsewhere in the literature and will not be treated here. However, note that Theorems 5.1 and 5.2 do describe the $E^{\infty}$ term of this spectral sequence.

As noted in the introduction, the first result (Theorem 5.1) generalizes a result of Kriz from [13], showing convergence of the Eilenberg-Moore cohomology spectral sequence for a fibration over $\mathrm{B}(\mathbb{Z} / p)$, with coefficients in Morava $K$-theory. The second result (Theorem 5.2) is the dual to a result of Dwyer [5] concerning convergence of the Eilenberg-Moore homology spectral sequence and in fact follows from it.

We start by recalling some known properties of fibrations and $\mathbb{S}$-algebras.

Lemma 5.5 Let $F \rightarrow E \rightarrow B$ be a fibration as above and let $R=k[\Omega B]$.

(1) $\operatorname{Hom}_{R}(k, k) \simeq \mathrm{C}^{*}(B)$ as $\mathbb{S}$-algebras.

(2) $\mathrm{C}_{*}(F) \otimes_{R} k \simeq \mathrm{C}_{*}(E)$.

(3) The modules $\operatorname{Hom}_{R}\left(k, \mathrm{C}^{*}(F)\right)$ and $\mathrm{C}^{*}(E)$ are equivalent as right $\mathrm{C}^{*}(B)-$ modules.

Proof First, recall that $\mathrm{W} \Omega B$ is a contractible free $\Omega B$-space. The term "free" is as defined by Rothenberg and Steenrod in [14]. It is easy to see that $\mathrm{C}_{*}(\mathrm{~W} \Omega B ; k)$ is a cell $R$-module (see [10, Definition III.2.1]) that is equivalent to $k$ as $R$-modules.

For the first equivalence, note that $\mathrm{W} \Omega B \times \Omega B$ pt is equivalent to the classifying space of $\Omega B$ which is $B$. Therefore, by [10, Proposition IV.7.5 and Theorem IV.7.8], we see $k \otimes_{R} k \simeq \mathrm{C}_{*}(B ; k)$. This equivalence implies:

$$
\begin{aligned}
\mathrm{C}^{*}(B) & \simeq \operatorname{Hom}_{k}\left(\mathrm{C}_{*}(B ; k), k\right) \simeq \operatorname{Hom}_{k}\left(k \otimes_{R} k, k\right) \\
& \simeq \operatorname{Hom}_{R}\left(k, \operatorname{Hom}_{k}(k, k)\right) \simeq \operatorname{Hom}_{R}(k, k)
\end{aligned}
$$


This equivalence is indeed an equivalence of $\mathbb{S}$-algebras. This was noted by Dwyer and Wilkerson in [9, Lemma 2.10] and also by Dwyer, Greenlees and Iyengar in [7, 4.22]. We sketch the argument here. Consider the following maps of $\mathbb{S}$-algebras, all of which are equivalences:

$$
\begin{aligned}
\mathrm{C}^{*}(B) & =\operatorname{Hom}_{k}\left(\mathrm{C}_{*}(B ; k), k\right) \cong \operatorname{Hom}_{k}\left(\mathrm{C}_{*}(\mathrm{pt} \times \Omega B \mathrm{~W} \Omega B), k\right) \\
& \cong \operatorname{Hom}_{R}\left(\mathrm{C}_{*}(\mathrm{~W} \Omega B), k\right) \stackrel{\simeq}{\longleftarrow} \operatorname{Hom}_{R}\left(\mathrm{C}_{*}(\mathrm{~W} \Omega B), \mathrm{C}_{*}(\mathrm{~W} \Omega B)\right)
\end{aligned}
$$

The first isomorphism follows from the structure of $\mathrm{W} \Omega B$ as a free $\Omega B$-space. The second isomorphism is [10, Proposition III.6.3].

For the second equivalence, recall the homotopy orbit space of the $\Omega B$-space $F$ is $F_{h \Omega B}=F \times_{\Omega B} \mathrm{~W} \Omega B$. There is a well known equivalence $E \simeq F_{h \Omega B}$ (this is sometimes called the Borel correspondence). Using again the results of [10, Proposition IV.7.5 and Theorem IV.7.8], we see that $\mathrm{C}_{*}(F) \otimes_{R} k \simeq \mathrm{C}_{*}(E)$ (we consider $F$ as a right $\Omega B$-space, thus $\mathrm{C}^{*}(F)$ becomes a left $R$-module).

This last equivalence implies that:

$$
\begin{aligned}
\operatorname{Hom}_{R}\left(k, \mathrm{C}^{*}(F)\right) & \simeq \operatorname{Hom}_{R}\left(k, \operatorname{Hom}_{k}\left(\mathrm{C}_{*}(F), k\right)\right) \\
& \simeq \operatorname{Hom}_{k}\left(\mathrm{C}_{*}(F) \otimes_{R} k, k\right) \simeq \mathrm{C}^{*}(E)
\end{aligned}
$$

Both $\operatorname{Hom}_{R}\left(k, \mathrm{C}^{*}(F)\right)$ and $\mathrm{C}^{*}(E)$ are right $\mathrm{C}^{*}(B)$-modules. By the composition pairing

$$
\operatorname{Hom}_{R}\left(k, \mathrm{C}^{*}(F)\right) \otimes_{k} \operatorname{Hom}_{R}(k, k) \rightarrow \operatorname{Hom}_{R}\left(k, \mathrm{C}^{*}(F)\right)
$$

(see [10, Proposition III.6.12]), the module $\operatorname{Hom}_{R}\left(k, \mathrm{C}^{*}(F)\right.$ ) is naturally a right $\operatorname{Hom}_{R}(k, k)$-module. The map $\mathrm{C}^{*}(B) \stackrel{\sim}{\rightarrow} \operatorname{Hom}_{R}(k, k)$ makes $\operatorname{Hom}_{R}\left(k, \mathrm{C}^{*}(F)\right)$ into a right $\mathrm{C}^{*}(B)$-module. For $\mathrm{C}^{*}(E)$, it is a right $\mathrm{C}^{*}(B)$-module by the map of $\mathbb{S}$ algebras $\mathrm{C}^{*}(B) \rightarrow \mathrm{C}^{*}(E)$.

The equivalence $\operatorname{Hom}_{R}\left(k, \mathrm{C}^{*}(F)\right) \simeq \mathrm{C}^{*}(E)$ is an equivalence of right $\mathrm{C}^{*}(B)$-modules. An outline of a proof of this fact, for the case $k=H \mathbb{F}_{p}$, can be found in [9], in the course of the proof of Lemma 2.10. These arguments of Dwyer and Wilkerson from [9] readily generalize to an arbitrary $\mathbb{S}$-algebra $k$, showing the modules $\operatorname{Hom}_{R}\left(k, \mathrm{C}^{*}(F)\right)$ and $C^{*}(E)$ are equivalent as right $C^{*}(B)$-modules.

As mentioned at the beginning of this section, results of Dwyer, Greenlees and Iyengar from [7] show, under some conditions, that the natural map $\mathrm{C}^{*}(E) \otimes_{\mathrm{C}^{*}(B)} k \rightarrow \mathrm{C}^{*}(F)$ is a $k$-cellular approximation of $\mathrm{C}^{*}(F)$ over $R$. These conditions are summarized in the following lemma. 
Lemma 5.6 If any one of the three conditions below holds, then $k$ is proxy-small as an $R$-module and the map $\mathrm{C}^{*}(E) \otimes_{\mathrm{C}^{*}(B)} k \rightarrow \mathrm{C}^{*}(F)$ (defined in $\mathbf{D}_{R}$ ) is a $k$-cellular approximation of $\mathrm{C}^{*}(F)$ as an $R$-module.

(1) $k$ is a commutative $\mathbb{S}$-algebra and $B$ is a finite space.

(2) $k$ is a commutative $\mathbb{S}$-algebra such that $\pi_{0}(k)$ is an $\mathbb{F}_{p}$ algebra and $B$ is equivalent to an Eilenberg-Mac Lane space of type $K(N, 1)$ where $N$ is a finite nilpotent group.

(3) $k$ is a commutative ring (in the classical sense) and $B \simeq K(G, 1)$, where $G$ is a finite group.

Proof We first explain the origin of the map $\mathrm{C}^{*}(E) \otimes_{\mathrm{C}^{*}(B)} k \rightarrow \mathrm{C}^{*}(F)$. By the previous lemma 5.5 there is an equivalence:

$$
\mathrm{C}^{*}(E) \otimes_{\mathrm{C}^{*}(B)} k \simeq \operatorname{Hom}_{R}\left(k, \mathrm{C}^{*}(F)\right) \otimes_{\mathrm{End}_{R}(k)} k
$$

This is an equivalence of $R$-modules, since the $R$-module structure on each of the tensor products is induced by the $R$-module structure of $k$. There is a natural map $\operatorname{Hom}_{R}\left(k, \mathrm{C}^{*}(F)\right) \otimes_{\mathrm{End}_{R}(k)} k \rightarrow \mathrm{C}^{*}(F)$ of $R$-modules (adjoint to the identity map id: $\operatorname{Hom}_{R}\left(k, \mathrm{C}^{*}(F)\right) \rightarrow \operatorname{Hom}_{R}\left(k, \mathrm{C}^{*}(F)\right)$ of right $\operatorname{End}_{R}(k)$-modules). This is the map referred to in the statement of the lemma.

If $k$ is proxy-small as an $R$-module, then the map $\operatorname{Hom}_{R}\left(k, \mathrm{C}^{*}(F)\right) \rightarrow \mathrm{C}^{*}(F)$ is a $k$-cellular approximation of $\mathrm{C}^{*}(F)$ as an $R$-module, by [7, Theorem 4.10]. Every one of the conditions above ensures $k$ is a proxy-small $R$-module. The first condition by [7, Proposition 5.3], the second condition by Proposition 4.7 and the third condition by [7, Example 5.9].

Example 5.7 We continue with Example 3.9. Recall $G$ is a finite group and $G \rightarrow$ $\mathrm{SU}(n)$ is an embedding of groups. As in Example 3.9 we take $k$ to be a commutative ring. Consider the fibration sequence:

$$
G \rightarrow \mathrm{SU}(n) \rightarrow \mathrm{SU}(n) / G
$$

The base space of this fibration is a finite space, thus satisfying the first condition of Lemma 5.6. Therefore the map

$$
\mathrm{C}^{*}(\mathrm{SU}(n)) \otimes_{\mathrm{C}^{*}(\mathrm{SU}(n) / G)} k \rightarrow k G
$$

is a $k$-cellular approximation of $R$-modules, with $R$ the group $\operatorname{ring} k[\Omega(\operatorname{SU}(n) / G)]$. In Example 3.9 we saw there is a strong independence of base (Lemma 3.1) with 
respect to the map $R \rightarrow k G$ and the module $k$. In particular $\operatorname{Cell}_{k}^{R}(k G)$ is equivalent to $\operatorname{Cell}^{G}(k G)$. We conclude that:

$$
\mathrm{C}^{*}(\mathrm{SU}(n)) \otimes_{\mathrm{C}^{*}(\mathrm{SU}(n) / G)} k \simeq \operatorname{Cell}^{G}(k G)
$$

This implies that the target of the Eilenberg-Moore cohomology spectral sequence for the fibration $G \rightarrow \mathrm{SU}(n) \rightarrow \mathrm{SU}(n) / G$ is Cell ${ }^{G} k G$. For example, if $G$ is an abelian group, then the results of Dwyer and Greenlees [6] imply that

$$
\pi_{-q}\left(\mathrm{C}^{*}(\mathrm{SU}(n)) \otimes_{\mathrm{C}^{*}(\mathrm{SU}(n) / G)} k\right) \cong H_{I}^{q}(k G)
$$

where $I$ is the augmentation ideal of $k G$ and $H_{I}^{*}(-)$ denotes the local cohomology groups with respect to $I$.

Before continuing to the proofs of Theorems 5.1 and 5.2, we demonstrate the use of cellular approximations by giving a different proof to the following result of Dwyer from [4].

Proposition 5.8 Let $k$ be a commutative ring and let $F \rightarrow E \rightarrow B$ be a fibration sequence where $E$ and $B$ are connected and $B$ is either a finite space or the classifying space of some finite group. Suppose $\pi_{1}(B)$ acts nilpotently on the cohomology groups $H^{n}(F ; k)$, ie for every $n \geq 0$ and $x \in H^{n}(F ; k)$ there exists some $m \geq 0$ such that $(1-g)^{m} x=0$ for all $g \in \pi_{1}(B)$. Then there is an equivalence of $k \Omega B$-modules:

$$
\mathrm{C}^{*}(E) \otimes_{\mathrm{C}^{*}(B)} k \simeq \mathrm{C}^{*}(F)
$$

Proof As usual, we use $R$ to denote the $\mathbb{S}$-algebra $k[\Omega B]$. From Lemma 5.6 we see it is sufficient to show that $\mathrm{C}^{*}(F)$ is a $k$-cellular $R$-module. Because $R$ is connective and $\mathrm{C}^{*}(F)$ is bounded above, $\mathrm{C}^{*}(F)$ is built from it's homotopy groups (see the proof of Proposition 2.7). In other words, $C^{*}(F)$ is built by the $k\left[\pi_{1}(B)\right]$-modules $\left\{H^{n}(F ; k)\right\}_{n \geq 0}$. So, it is enough to show that for every $n, H^{n}(F ; k)$ is a $k$-cellular $R$-module.

Fix some $n$. The $k\left[\pi_{1}(B)\right]$-module $H^{n}(F ; k)$ can be written as an increasing union of nilpotent $k\left[\pi_{1}(B)\right]$-modules: $H^{n}(F ; k)=\bigcup_{i \geq 1} N_{i}$ where $N_{i}$ is a nilpotent $k\left[\pi_{1}(B)\right]-$ module of class $i$. It is easy to see, using induction on $i$, that each $N_{i}$ is a $k$-cellular $k\left[\pi_{1}(B)\right]$-module. The $k\left[\pi_{1}(B)\right]$-module $H^{n}(F ; k)$ is equivalent to the homotopy colimit of the telescope $N_{1} \rightarrow N_{2} \rightarrow \cdots$. Since the homotopy colimit of $k$-cellular modules is $k$-cellular (see, for example Dror Farjoun [3]), we conclude $H^{n}(F ; k)$ is $k$-cellular as a $k\left[\pi_{1}(B)\right]$-module. But this implies $H^{n}(F ; k)$ is $k$-cellular also as an $R$-module. 
Proof of Theorem 5.1 By [10, Theorem III.4.2], the equivalence $\Omega B \simeq N$ induces an equivalence of the derived categories: $\mathbf{D}_{R} \simeq \mathbf{D}_{k N}$. This means we can work in the category of $k N$-modules instead of $R$-modules. To do that we replace $F$ by an equivalent $N$-space, which we will also denote $F$. Since $\mathrm{C}^{*}(E) \otimes_{\mathrm{C}^{*}(B)} k \simeq$ Cell $^{N} \mathrm{C}^{*}(F)$, the result follows from Proposition 4.4 by noting that:

$$
\operatorname{Hom}_{k[H]}\left(k, \mathrm{C}^{*}(F)\right) \simeq \operatorname{Hom}_{k}\left(\mathrm{C}_{*}(F) \otimes_{k[H]} k, k\right) \simeq \mathrm{C}^{*}\left(F_{h(H)}\right)
$$

The last equivalence: $\mathrm{C}_{*}(F) \otimes_{k[H]} k \simeq F_{h(H)}$, follows from two results of Elmendorf, Kriz, Mandell and May [10, Proposition IV.7.5 and Theorem IV.7.8].

Our next goal is to prove Theorem 5.2. For that purpose, we will decompose the topological group $\Omega B$ into the following homotopy fibration sequence

$$
\Omega \widetilde{B} \rightarrow \Omega B \rightarrow \Omega K\left(\pi_{1}(B), 1\right)
$$

where $\widetilde{B}$ is the universal cover of $B$ and $K\left(\pi_{1}(B), 1\right)$ is the appropriate EilenbergMac Lane space (ie the classifying space of $\pi_{1}(B)$ ).

Let $Q$ denote the topological group $\Omega K\left(\pi_{1}(B), 1\right)$ and set $G=\pi_{1}(B)$. Instead of working with $k Q$-modules, we would much rather work with $k G$-modules. The following observation shows that for all of our purposes we can replace $k Q$-modules by $k G$-modules. The map $\mu: Q \rightarrow \pi_{0}(Q)=G$ is a weak equivalence and therefore the induced map $\mu: \mathrm{C}_{*}(Q ; k) \rightarrow k G$ of $\mathbb{S}$-algebras is also a weak equivalence. By [10, Theorem III.4.2], the functor $\mu^{*}: \mathbf{D}_{k G} \rightarrow \mathbf{D}_{k Q}$, induced by $\mu$, is an equivalence of these derived categories. This implies we can work over $k G$ instead of $k Q$. Moreover, the map $\varphi: R \rightarrow k G$, induced by the map $\Omega B \rightarrow \pi_{0}(\Omega B)=G$, is equal to the composition $R \stackrel{\psi}{\rightarrow} k Q \stackrel{\mu}{\rightarrow} k G$. Hence the functors: $\psi^{*} \mu^{*}$ and $\varphi^{*}$ are equal.

Here is the main idea of the proof of Theorem 5.2. Recall $F \rightarrow E \rightarrow B$ is a fibration with $B$ a finite, connected, nilpotent space and $k$ is the field $\mathbb{F}_{p}$. From the proof of Proposition 2.7 we see that $C^{*}(F)$ is built from the $k\left[\pi_{1}(B)\right]$-modules $\left\{H^{n}(F ; k)\right\}_{n \geq 0}$. We will show that the $k$-cellular approximation of $\mathrm{C}^{*}(F)$ (which is $\mathrm{C}^{*}(E) \otimes_{\mathrm{C}^{*}(B)} k$ ) is built from the $k\left[\pi_{1}(B)\right]$-modules: $\left\{\operatorname{Cell}_{1}^{\pi}(B) H^{n}(F ; k)\right\}_{n \geq 0}$. Then we apply the results of Section 4 to compute the modules $\operatorname{Cell}^{\pi_{1}(B)} H^{n}(F ; k)$.

Proof of Theorem 5.2 Set $k=\mathbb{F}_{p}$, we remind the reader that $R$ denotes the group ring $k[\Omega B], N$ is the fundamental group $\pi_{1}(B), P$ is a $p$-Sylow subgroup of $N$ and $H=N / P$. By Lemma 5.6, $\mathrm{C}^{*}(E) \otimes_{\mathrm{C}^{*}(B)} k$ is a $k$-cellular approximation of $\mathrm{C}^{*}(F)$ as an $R$-module. So it is enough to show $\operatorname{Cell}_{k}^{R} \mathrm{C}^{*}(F)$ has the desired homotopy groups. 
Example 2.9 shows that $\mathrm{C}_{*}(\widetilde{B})$ is a $k$-cellular $k N$-module. So by Proposition 3.8, for every $N$-module $X$, the map $\mathrm{Cell}^{N} X \rightarrow X$ is a $k$-cellular approximation of $X$ as an $R$-module. Proposition 4.4 shows that the map $\operatorname{Hom}_{k N}(k P, X) \rightarrow X$ is a $k$-cellular approximation of $X$ as a $k N$-module. In fact, as noted in Remark 4.5, $\operatorname{Hom}_{k N}(k P, X)$ is the strict fixed points of the $H$ action on $X$, denoted $X^{H}$.

We use now the notation of Dwyer and Greenlees from [6], this is the same notation used in the proof of Proposition 2.7. In this notation $H^{n}(F ; k) \cong \mathrm{C}^{*}(F)\langle-n,-n\rangle$ and $\operatorname{Cell}_{k}^{R}\left(\mathrm{C}^{*}(F)\langle-n,-n\rangle\right) \simeq H^{n}(F ; k)^{H}$. We now prove, by induction on $i$, that for every $n$ such that $0 \leq n \leq i$ :

$$
\pi_{-n} \operatorname{Cell}_{k}^{R}\left(\mathrm{C}^{*}(F)\langle-i, 0\rangle\right) \cong H^{n}(F ; k)^{H}
$$

For the induction step, consider the triangle:

$$
\mathrm{C}^{*}(F)\langle-i, 0\rangle \rightarrow \mathrm{C}^{*}(F)\langle-i-1,0\rangle \rightarrow \mathrm{C}^{*}(F)\langle-i-1,-i-1\rangle
$$

Taking $k$-cellular approximation of this triangle yields the following triangle:

$\operatorname{Cell}_{k}^{R}\left(\mathrm{C}^{*}(F)\langle-i, 0\rangle\right) \rightarrow \operatorname{Cell}_{k}^{R}\left(\mathrm{C}^{*}(F)\langle-i-1,0\rangle\right) \rightarrow \operatorname{Cell}_{k}^{R}\left(\mathrm{C}^{*}(F)\langle-i-1,-i-1\rangle\right)$

The long exact sequence of homotopy groups, for this last triangle, shows that (1) holds for $0 \leq n \leq i+1$, thus completing the induction.

From the proof of Proposition 2.7 we see that: $\mathrm{C}^{*}(F) \simeq \operatorname{hocolim}_{i} \mathrm{C}^{*}(F)\langle-i, 0\rangle$. Next we show that:

$$
\operatorname{Cell}_{k}^{R} \mathrm{C}^{*}(F) \simeq \operatorname{hocolim}_{i} \operatorname{Cell}_{k}^{R}\left(\mathrm{C}^{*}(F)\langle-i, 0\rangle\right)
$$

By a result of Dwyer and Greenlees [6, Proposition 4.3], the $k$-cellular approximation of an $R$-module $X$ is given by the map $\operatorname{Cell}_{k}^{R}(R) \otimes_{R} X \rightarrow X$, whenever $k$ is proxysmall. The functor $\operatorname{Cell}_{k}^{R}(R) \otimes_{R}-$ is a left adjoint and therefore commutes with homotopy colimits. Hence taking $k$-cellular approximations commutes with homotopy colimits and the desired equivalence in (2) follows.

Finally, from (1) and (2) above it easy to see that:

$$
\pi_{-n}\left(\operatorname{Cell}_{k}^{R} \mathrm{C}^{*}(F ; k)\right) \cong \pi_{-n}\left(\operatorname{Cell}_{k}^{R}\left(\mathrm{C}^{*}(F ; k)\langle-n, 0\rangle\right)\right) \cong H^{n}(F ; k)^{H}
$$

\section{A spectral sequence}

As before, let $F \rightarrow E \rightarrow B$ be a fibration sequence. The proof of Theorem 5.2 shows that when $B$ is a finite nilpotent space and $k$ is a commutative ring then $\operatorname{Cell}_{k}^{R} \mathrm{C}^{*}(F)$ is built from the modules $\operatorname{Cell}_{k}^{k\left[\pi_{1}(B)\right]} H^{n}(F ; k)$. We use this observation to construct 
a spectral sequence converging (conditionally) to $\operatorname{Cell}_{k}^{R} \mathrm{C}^{*}(F ; k)$, where $k$ is any commutative ring. Below, in Section 6.1, we demonstrate the use of this spectral sequence for the case where $\pi_{1}(B)$ is a cyclic group.

Proposition 6.1 Let $F \rightarrow E \rightarrow B$ be a fibration where $E$ and $B$ are connected and let $k$ be a commutative ring. Suppose $B$ is a finite nilpotent space and set $N=\pi_{1}(B)$. There exists a spectral sequence

$$
E_{p, q}^{1}=\pi_{2 p+q}\left(\operatorname{Cell}^{N} H^{p}(F ; k)\right) \Rightarrow \pi_{p+q}\left(\operatorname{Cell}_{k}^{R} \mathrm{C}^{*}(F ; k)\right)
$$

where $R$ denotes the $\mathbb{S}$-algebra $k[\Omega B]$. Convergence is as in the usual case for the spectral sequence of the homotopy colimit of spectra.

Proof The $\mathbb{S}$-algebra $R=k[\Omega B]$ is connective and bounded-above. Connectivity of $R$ implies the existence of Postnikov sections in the category of $R$-modules (see [7, Lemma 3.2]). Since $k$ is bounded above, the $R$-module $C^{*}(F)$ is also bounded above. So, the same arguments as in the proof of Theorem 5.2 show that:

$$
\operatorname{Cell}_{k}^{R} \mathrm{C}^{*}(F) \simeq \operatorname{hocolim}_{p} \operatorname{Cell}_{k}^{R}\left(\mathrm{C}^{*}(F)\langle-p, 0\rangle\right)
$$

This gives a spectral sequence whose $E^{1}$-term is:

$$
E_{p, q}^{1}=\pi_{p+q}\left(\operatorname{Cone}\left(\operatorname{Cell}_{k}^{R} \mathrm{C}^{*}(F)\langle-p+1,0\rangle \rightarrow \operatorname{Cell}_{k}^{R} \mathrm{C}^{*}(F)\langle-p, 0\rangle\right)\right)
$$

For $p=0$, we get: $E_{0, q}^{1}=\pi_{q}\left(\operatorname{Cell}_{k}^{R} \mathrm{C}^{*}(F)\langle 0,0\rangle\right)$. For $p>0$, note that taking $k$-cellular approximations preserves triangles. Hence:

$$
\begin{aligned}
\operatorname{Cone}\left(\operatorname{Cell}_{k}^{R} \mathrm{C}^{*}(F)\langle-p+1,0\rangle\right. & \left.\rightarrow \operatorname{Cell}_{k}^{R} \mathrm{C}^{*}(F)\langle-p, 0\rangle\right) \\
& \simeq \operatorname{Cell}_{k}^{R} \operatorname{Cone}\left(\mathrm{C}^{*}(F)\langle-p+1,0\rangle \rightarrow \mathrm{C}^{*}(F)\langle-p, 0\rangle\right) \\
& \simeq \operatorname{Cell}_{k}^{R}\left(\Sigma^{-p} H^{p}(F ; k)\right)
\end{aligned}
$$

The same arguments as in the proof of Theorem 5.2 show that

$$
\mathrm{Cell}_{k}^{R} H^{p}(F ; k) \simeq \operatorname{Cell}^{N} H^{p}(F ; k)
$$

for every $p$. This yields the desired $E^{1}$-page.

Remark 6.2 The proposition above holds also when $k$ is a commutative, connective, bounded above $\mathbb{S}$-algebra. 


\subsection{Fibrations over a nilpotent space with cyclic fundamental group}

The setting we consider is as follows. Let $F \rightarrow E \rightarrow B$ be a homotopy fibration sequence. We assume $B$ is a connected, finite, nilpotent space with a cyclic fundamental group and $E$ is a connected space. Let $k$ be a Noetherian commutative ring. We use $C$ to denote the fundamental group $\pi_{1}(B)$.

As mentioned earlier, a result of Dwyer and Greenlees [6] connects $k$-cellular approximations over $k C$ with $I$-local cohomology of $k C$-modules, where $I$ is the augmentation ideal of $k C$. This gives the following explicit description for $k$-cellular approximations over $k C$. Let $x$ be a generator of $C$, so $z=(1-x)$ is a generator of $I$. Then, for every $k C$-chain complex $M$, there is a triangle:

$$
\mathrm{Cell}^{C} M \rightarrow M \rightarrow M[1 / z]
$$

where $M[1 / z]$ is the homotopy colimit of the telescope $M \stackrel{z}{\rightarrow} M \stackrel{z}{\rightarrow} \cdots$. Note that this implies $\left(\pi_{i} M\right)[1 / z]=\pi_{i}(M[1 / z])$. In particular, we have a long exact sequence:

$$
\cdots \rightarrow \pi_{i} \mathrm{Cell}^{C} M \rightarrow \pi_{i} M \rightarrow \pi_{i} M[1 / z] \rightarrow \pi_{i-1} \mathrm{Cell}^{C} M \rightarrow \cdots
$$

We shall use the notation $\Gamma_{I} M$ for the $I$-power torsion sub-chain complex of $M$, namely:

$$
\Gamma_{I} M=\left\{m \in M \mid I^{n} m=0 \text { for some } n\right\}
$$

So, if $\pi_{i}(M)=0$ for all $i \neq 0$ and $\pi_{0}(M)=M_{0}$ we have the following exact sequence:

$$
0 \rightarrow \pi_{0} \mathrm{Cell}^{C} M \rightarrow M_{0} \rightarrow M_{0}[1 / z] \rightarrow \pi_{-1} \mathrm{Cell}^{C} M \rightarrow 0
$$

This implies

$$
\pi_{0} \mathrm{Cell}^{C} M=\Gamma_{I} M_{0}, \quad \pi_{-1} \mathrm{Cell}^{C} M=\left(M_{0}[1 / z]\right) / M_{0}
$$

and $\pi_{i}$ Cell ${ }^{C} M=0$ for $i \neq 0,-1$. We have gathered all the ingredients to prove the following result.

Lemma 6.3 Let $F \rightarrow E \rightarrow B$ be a homotopy fibration sequence. Suppose $B$ and $E$ are connected and $B$ is a finite, nilpotent space with a cyclic fundamental group with generator $x$. Let $k$ be a Noetherian commutative ring and let $I$ denote the augmentation ideal of the group ring $k\left[\pi_{1}(B)\right]$. Then

$$
\pi_{0}\left(\mathrm{C}^{*}(E) \otimes_{\mathrm{C}^{*}(B)} k\right)=\Gamma_{I} H^{0}(F ; k)
$$

and for every $n>0$ there is a short exact sequence

$$
0 \rightarrow H^{n-1}(F ; k)[1 / z] / H^{n-1}(F ; k) \rightarrow \pi_{-n}\left(\mathrm{C}^{*}(E) \otimes_{\mathrm{C}^{*}(B)} k\right) \rightarrow \Gamma_{I} H^{n}(F ; k) \rightarrow 0
$$

where $z=1-x$. 
Proof Since $B$ is a finite space, by Lemma 5.6 we see that $C^{*}(E) \otimes_{\mathrm{C}^{*}(B)} k \simeq$ $\mathrm{Cell}_{k}^{k[\Omega B]} \mathrm{C}^{*}(F)$. Now we apply the spectral sequence of Proposition 6.1. Note that the homotopy groups of $\mathrm{Cell}^{C} H^{p}(F ; k)$ are: $\Gamma_{I} H^{p}(F ; k)$ in dimension 0 , $H^{p}(F ; k)[1 / z] / H^{p}(F ; k)$ in dimension -1 and zero elsewhere. Using standard spectral sequence arguments we obtain the desired result.

Here is an example for the use of this lemma.

Corollary 6.4 Let $F, E$ and $B$ be as in Lemma 6.3. Suppose in addition that $\pi_{1}(B)$ is the cyclic group of order 2 and $F$ is of finite type. If $\mathrm{C}^{*}(E ; \mathbb{Z}) \otimes_{\mathrm{C}^{*}(B ; \mathbb{Z})} \mathbb{Z} \simeq \mathbb{Z}$ then for every $n>0, H^{n}(F ; \mathbb{Z})$ are finite groups of odd order with the action of $\pi_{1}(B)$ being multiplication by -1 , and $H^{0}(F ; \mathbb{Z})=\mathbb{Z}$ has the trivial action of $\pi_{1}(B)$.

Proof Denote by $x$ the generator of $\pi_{1}(B)$ and set $z=1-x \in \mathbb{Z}\left[\pi_{1}(B)\right]$. Since $z^{2}=2 z$, then for every discrete $\mathbb{Z}\left[\pi_{1}(B)\right]$-module $M$, the discrete module $M[1 / z]$ is uniquely divisible by 2 . From Lemma 6.3 we see that for $n>0, H^{n}(F ; \mathbb{Z}) \cong$ $H^{n}(F ; \mathbb{Z})[1 / z]$ and in particular $\Gamma_{I} H^{n}(F ; \mathbb{Z})=0$. Hence $H^{n}(F ; \mathbb{Z})$ is a finitely generated abelian group that is uniquely divisible by 2 , and therefore must have odd order. For any element $a \in H^{n}(F ; \mathbb{Z})$ the element $x \cdot a+a$ is in $\Gamma_{I} H^{n}(F ; \mathbb{Z})$, therefore $x \cdot a=-a$.

For $H^{0}(F ; \mathbb{Z})$, note that it is isomorphic to the finitely generated free abelian group $\mathbb{Z} \pi_{0}(F)=\bigoplus_{a \in \pi_{0}(F)} \mathbb{Z}$ with the $\pi_{1}(B)$ action coming from the action of $\pi_{1}(B)$ on $\pi_{0}(F)$. Suppose the action of $\pi_{1}(B)$ on $\pi_{0}(F)$ had a free orbit. Then $H^{0}(F ; \mathbb{Z})$ would have a direct summand isomorphic to $\mathbb{Z}\left[\pi_{1}(B)\right]$. However, the map $\mathbb{Z}\left[\pi_{1}(B)\right] \rightarrow$ $\mathbb{Z}\left[\pi_{1}(B)\right][1 / z]$ cannot be a surjection, because $\mathbb{Z}\left[\pi_{1}(B)\right]$ is not a 2 -divisible group. Hence the action of $\pi_{1}(B)$ on $\pi_{0}(F)$ is trivial and the result follows.

\section{References}

[1] A K Bousfield, The localization of spectra with respect to homology, Topology 18 (1979) 257-281 MR551009

[2] W Chachólski, On the functors $C W_{A}$ and $P_{A}$, Duke Math. J. 84 (1996) 599-631 MR1408539

[3] E Dror Farjoun, Cellular spaces, null spaces and homotopy localization, Lecture Notes in Math. 1622, Springer, Berlin (1996) MR1392221

[4] W G Dwyer, Strong convergence of the Eilenberg-Moore spectral sequence, Topology 13 (1974) 255-265 MR0394663

[5] W G Dwyer, Exotic convergence of the Eilenberg-Moore spectral sequence, Illinois J. Math. 19 (1975) 607-617 MR0383409 
[6] W G Dwyer, J P C Greenlees, Complete modules and torsion modules, Amer. J. Math. 124 (2002) 199-220 MR1879003

[7] W G Dwyer, J P C Greenlees, S Iyengar, Duality in algebra and topology, Adv. Math. 200 (2006) 357-402 MR2200850

[8] W G Dwyer, J P C Greenlees, S Iyengar, Finiteness in derived categories of local rings, Comment. Math. Helv. 81 (2006) 383-432 MR2225632

[9] W G Dwyer, C W Wilkerson, The fundamental group of a p-compact group, to appear in Bull. Lond. Math. Soc.

[10] A D Elmendorf, I Kriz, M A Mandell, J P May, Rings, modules, and algebras in stable homotopy theory, Math. Surveys and Monogr. 47, Amer. Math. Soc. (1997) MR1417719 With an appendix by M Cole

[11] P Hilton, G Mislin, J Roitberg, Localization of nilpotent groups and spaces, NorthHolland Math. Stud. 15, North-Holland, Amsterdam (1975) MR0478146

[12] PS Hirschhorn, Model categories and their localizations, Math. Surveys and Monogr. 99, Amer. Math. Soc. (2003) MR1944041

[13] I Kriz, Morava K-theory of classifying spaces: some calculations, Topology 36 (1997) 1247-1273 MR1452850

[14] M Rothenberg, N E Steenrod, The cohomology of classifying spaces of $H$-spaces, Bull. Amer. Math. Soc. 71 (1965) 872-875 MR0208596

[15] S Schwede, B Shipley, Stable model categories are categories of modules, Topology 42 (2003) 103-153 MR1928647

School of Mathematics and Statistics, The University of Sheffield Sheffield, S3 7RH, UK

s.shamir@sheffield.ac.uk

Received: 13 December 2007 Revised: 17 April 2009 